\title{
Numerical Investigation of the Fluid-Solid Mixture Flow using the FOCUS Code
}

\author{
Kailun Guo, Ronghua Chen*, Yonglin Li, Suizheng Qiu, G.H. Su
}

School of Nuclear Science and Technology, State Key Laboratory of Multiphase Flow in

Power Engineering, Xi'an Jiaotong University, No. 28, Xianning West road, Xi'an 710049,

China

*Corresponding authors: Email: rhchen@mail.xjtu.edu.cn ; Tel \& Fax: +86-029- 82667987 


\begin{abstract}
The fluid-solid mixture flow is the key phenomenon in the reactor core when severe accident happens, there are both fluid phase and solid phase in the migration of the molten corium. In this study, the discrete element method (DEM) is applied to model the interaction between solid bodies, the MPS method is adopted to simulate the fluid flows, and these two methods are coupled by the model of the solid and fluid phase interaction. The passively moving solid (PMS) model is adopted to modify the movement of moving particles so that several particles can form one solid body. The time step in the DEM model is different from that in the MPS method, thus a multiple time-step using inner iteration is carried out to solve this problem. The FOCUS (Fluid flOw CoUpling with Solid's movement) code is developed by the models and methods above using $\mathrm{C}_{++}$language. The simulations and experiments about collapse of solid cylinder layers and he breaking of a water dam involving solid cylinder layers in two-dimension are carried out to validate FOCUS, and a simple three-dimensional case is also tested before the future work about severe accidents in reactor cores. The comparisons between calculation results and experimental data are in good agreements with the average relative error less than $8.4 \%$, meaning the FOCUS is capable of simulating the fluid-solid mixture flow. It provides the foundation for the further work on simulations of the severe accidents in nuclear reactor cores.
\end{abstract}

Key words: Severe accident; MPS method; DEM model; Fluid-solid mixture flow; FOCUS code; Validations 


\section{Nomenclature}

$\vec{u} \quad$ particle velocity vector

$\vec{f} \quad$ forces between particles

$p \quad$ pressure, $\mathrm{Pa}$

$d \quad$ number of dimensions

$n \quad$ particle number density

$w \quad$ weight function

$r \quad$ position of particle(solid bodies)/ contact radius, $\mathrm{m}$

$t$ time, $s$

A coefficient matrix of the Poisson equation

$b$ source term of the Poisson equation

I moment of inertia

$N \quad$ number of particles

a acceleration

$F \quad$ collision force, $\mathrm{N}$

$T \quad$ moment, $\mathrm{N} \cdot \mathrm{m}$

$M \quad$ transition matrix

$X, Y, Z \quad$ local coordinates/displacement in each direction

$x, y, z \quad$ global coordinate/position in each direction

$D \quad$ Distance, $\mathrm{m}$

e elastic force, $\mathrm{N}$

$d$ damping force, $\mathrm{N}$

$k$ elastic coefficient, $\mathrm{N} / \mathrm{m}$

c damping coefficient, $\mathrm{N} / \mathrm{m}$

E elastic modulus

$R \quad$ radius of solid body

$v \quad$ Poisson ratio

$m \quad$ mass, $\mathrm{kg}$

$H \quad$ Initial height of solid layers, $\mathrm{m}$

$L_{1} \quad$ initial length between the right-most cylinder and the left wall of container, $\mathrm{m}$

$L_{2} \quad$ initial length of solid layers, $m$

\section{Greek letters}

$\begin{array}{ll}\rho & \text { density, } \mathrm{kg} / \mathrm{m}^{3} \\ \mu & \text { viscosity coefficient/fraction coefficient } \\ \phi & \text { physical quantity } \\ \lambda & \text { coefficient in MPS method } \\ \omega & \text { angular velocity, } \mathrm{rad} / \mathrm{s} \\ \alpha & \text { angular acceleration, } \mathrm{rad} / \mathrm{s}^{2}\end{array}$


$\theta \quad$ rotation angle, rad

\section{Superscript/subscript}

$\begin{array}{ll}i & \text { particle's number } \\ j & \text { neighbor particle's number } \\ i i & \text { solid body's number } \\ j j & \text { neighbor solid body's number } \\ l & \text { liquid phase } \\ S & \text { solid phase } \\ l s & \text { interactions between liquid and solid phase } \\ k & \text { number of time steps in DEM calculation } \\ c o l & \text { joint physical quantity } \\ M P S & \text { physical quantity in MPS calculation } \\ D E M & \text { physical quantity in DEM calculation } \\ \text { min } & \text { minimum } \\ n & \text { normal direction/ number of time steps in MPS calculation } \\ e & \text { tangent direction } \\ 0 & \text { initial condition } \\ * & \text { temporal }\end{array}$




\section{Introduction}

The moving particle semi-implicit (MPS) method (Koshizuka, 2010) is developing recent years, and it is one of the particle methods in which continuum mechanics is analyzed using moving particles. Since the MPS method is a meshless, fully Lagrangian, particle method, it has advantages in simulating many kinds of complex thermal-hydraulic problems in nuclear engineering, especially those with free surface. Recent years, the MPS method has been developed a lot, in gas-liquid two-phase flow simulation, Yoon developed MPS-MAFL which is an Eulerian-Lagrangian method to simulate the bubble growth in boiling condition ( Yoon H Y, Koshizuka S, Oka Y., 2001). Chen et al. applied this method into many other bubble dynamics problems, coalescence of bubble pairs rising in a stagnant liquid (R.H. Chen et al., 2011), bubble dynamics during flow boiling (R.H. Chen et al., 2010), etc. Duan developed the Contoured Continuum Surface Force model (CCFS) to simulate the surface tension force between two phases (G Duan et al., 2015). In the fluid-solid mixture flow simulation with MPS method, Zhang tried to coupling the DEM model with the MPS method (Zhang, S., Morita, K., et al., 2009), but his work lacked of quantitative analysis and the simulation results were not very satisfied. What's more, his work didn't spread to three-dimension. Besides the fluid dynamics, the energy equations are added in to the MPS method so that it can be applied to simulate severe accidents such as freezing controlled penetration behavior of the molten core debris in an instrument tube (R.H. Chen, Y. Oka et al., 2014a), melt freezing behavior in a tube (R.H. Chen, Y. Oka et al., 2014b). The MPS method is also used in other engineering fields: ship engineering, micro-flow, etc.

In engineering practice, the problems of balance and movement about the discontinuous granular bodies are always need to be solved, such as the movement of the fragments in earthquake, material transportation by conveyor belt, the fragmentation of fuel pellets when severe accident happens in nuclear power stations etc. Although the MPS method has many advantages in solving problems on continuum mechanics, it is not suitable in these kinds of problems any more.

In present research, a well-known fully Lagrangian method which is used to solve the 
problem of movement about the discontinuous granular bodies called discrete element method (DEM) is introduced. The DEM model is a numerical method which is utilized to analyzed the mechanical behavior of discontinuous granular bodies, the basic principle of this method is dividing the discontinuous granular bodies into discrete elements, and establishing equations of motion by Newton's second law for each element, then solving these equations by dynamic relaxation (DR) method to get the velocity and position of each discontinuous granular bodies. The DEM is firstly introduced by $P$.A .Cundall in 1971 (Cundall P A., 1971), after 40 years of development, this method has successfully used in many engineering fields.

In this study, a three-dimensional DEM model is developed based on the MPS code and coupled with the MPS method so that the new code named FOCUS (Fluid flOw CoUpling with Solid's movement) can solve the problem on fluid-solid mixture flow. The fluid-solid mixture flow is the key phenomenon in the reactor core when severe accident happens. During a severe accident in light water reactors, the continuous melting of the core may lead to the relocation of the corium melt into the lower plenum of the reactor vessel (Zhang et al., 2015a; Zhang et al., 2015b). The accumulated molten core material can form a hemispherical pool in the lower head with internal decay heating, which will gradually heat up the vessel wall and threaten the reactor vessel integrity (Zhang et al., 2016a; Zhang et al., 2016b), there are both fluid phase and solid phase in the migration of the molten corium. The FOCUS code can be utilized to simulated the whole process of the core melting in nuclear reactor.

\section{Theoretical Models}

\subsection{Models in MPS}

The governing equations for MPS method are the mass and momentum conservation equations (Koshizuka, 2010) as follows:

$$
\begin{gathered}
\frac{D \rho_{l}}{D t}+\rho_{l} \nabla \cdot \vec{u}=0 \\
\rho_{l} \frac{D \vec{u}}{D t}=-\nabla p+\mu \nabla^{2} \vec{u}+\rho_{l} \vec{f}_{l}
\end{gathered}
$$


where $\vec{u}, p$ and $\rho_{l}$ are the velocity, pressure and density of the fluid, respectively, $\mu$ is the viscosity coefficient, $\nabla p$ and $\mu \nabla^{2} \vec{u}$ are the pressure and viscosity forces within fluid phase, respectively, $\vec{f}_{l}$ is the forces between fluid particles.

To solve the governing equations in MPS method, the particle interaction models are developed.

The gradient model which is adopted to solve the pressure gradient term in MPS (Koshizuka, 2010) is as follows:

$$
\langle\nabla \phi\rangle_{i}=\frac{d}{n^{0}} \sum_{j \neq i} \frac{\phi_{j}-\phi_{i}^{\prime}}{\left|\vec{r}_{j}-\vec{r}\right|^{2}}\left(\vec{r}_{j}-\vec{r}_{i}\right) w\left(\left|\vec{r}_{j}-\vec{r}_{i}\right|\right)
$$

where $\phi$ is the physical quantity of particles, $d$ is the number of dimensions of the problem, $\vec{r}$ is the position of particles, $n^{0}$ is the constant particle number density, $w$ is the kernel function in MPS method, and the most classical kernel function that suggested by Koshizuka (Koshizuka, 2010) is as follows:

$$
w(r)= \begin{cases}\frac{r_{e}}{r}-1 & \left(r<r_{e}\right) \\ 0 & \left(r \geq r_{e}\right)\end{cases}
$$

where $r_{e}$ is the interaction radius of particles.

The Laplacian operator in MPS is formulated as:

$$
\left\langle\nabla^{2} \phi\right\rangle_{i}=\frac{2 d}{n^{0} \lambda} \sum_{j \neq i}\left(\phi_{j}-\phi_{i}\right) w\left(\left|\vec{r}_{j}-\vec{r}_{i}\right|\right)
$$

This model is developed to solve the viscosity term in momentum conservation equations, where $\lambda$ is a coefficient that calculated as follows:

$$
\lambda=\frac{\sum_{j \neq i} w\left(\mid \vec{r}_{j}-\vec{r}_{i}\right)\left|\vec{r}_{j}-\vec{r}_{i}\right|^{2}}{\sum_{j \neq i} w\left(\mid \vec{r}_{j}-\vec{r}_{i}\right)}
$$

The Poisson equation of pressure (Koshizuka, 2010) which is adopted to correct the velocity and position of particles is as follows: 


$$
\left\langle\nabla^{2} p^{n+1}\right\rangle_{i}=\frac{\rho}{\Delta t_{M P S}^{2}} \frac{n^{0}-\left\langle n^{*}\right\rangle_{i}}{n^{0}}
$$

where $\Delta t_{M P S}$ is the calculation time step in MPS method.

After introducing the particle interaction models, the governing equations can be discretized through these models. The pressure gradient term in momentum equation can be discretized by equation (3) as follows:

$$
\left\langle\frac{1}{\rho} \nabla P\right\rangle=\frac{1}{\rho_{i}} \frac{d}{n_{0}} \sum_{j \neq i}\left\{\frac{\left(P_{j}-P_{i, \min }\right)\left(\vec{r}_{j}-\vec{r}_{i}\right)}{\left|\vec{r}_{j}-\vec{r}_{i}\right|^{2}} w\left(\left|\vec{r}_{j}-\vec{r}_{i}\right|\right)\right\}
$$

where $P_{i, \text { min }}$ is the minimum pressure among neighbor particles, it is used to keep the stabilizing of the calculation (Koshizuka S, Nobe A, Oka Y, 1998).

The viscosity term in momentum conservation equations can be discretized by equation (5) as follows:

$$
\mu_{i}\left\langle\nabla^{2} \vec{u}\right\rangle_{i}=\frac{2 d}{n^{0} \lambda} \mu_{i} \sum_{j \neq i}\left(\vec{u}_{j}-\vec{u}_{i}\right) w\left(\left|\vec{r}_{j}-\vec{r}_{i}\right|\right)
$$

Equations (8) and (9) are solved in an explicit way in MPS method.

The left side of equation (7) can also be discretized by equation (5):

$$
\left\langle\nabla^{2} p\right\rangle_{i}=\frac{2 d}{n^{0} \lambda} \sum_{j \neq i}\left[\left(p_{j}-p_{i}\right) w\left(\left|\vec{r}_{j}-\vec{r}_{i}\right|\right)\right]
$$

equation (7) can be rewritten as the form like $A p=b$, where A is the coefficient matrix of the Poisson equation, and $b$ is the source term of it. Then the equations are solved by implicit iterative method.

\subsection{DEM model}

The MPS method is a meshless numerical method which analyzes the continuum mechanics by the concept of moving particles, and the DEM is a method to solve the movement of the discontinuous granular bodies, there are something in common between these two methods. Both in DEM and MPS, the solid material are considered as rigid body, and the plastic deformation is not considered. It is remarkable that the size of the solid 
body in the DEM method is always larger than the particle's average distance in the MPS method. When compiling the DEM model based on MPS code, how to make several solid particles form one solid body is the first problem to be solved. The equations of initializing the velocity of the solid body using the passively moving solid (PMS) model (Koshizuka, S., Nobe, A. et al., 1998) are formulated as follows:

$$
\left\{\begin{array}{l}
\overrightarrow{\boldsymbol{u}}_{i i}^{k}=\frac{1}{N} \sum_{i=1}^{N} \overrightarrow{\boldsymbol{u}}_{i}^{k} \\
\overrightarrow{\boldsymbol{\omega}}_{i i}^{k}=\frac{1}{\boldsymbol{I}_{i i}} \sum_{i=1}^{N} \boldsymbol{m}_{i}\left(\vec{r}_{i}^{k}-\vec{r}_{i i}^{k}\right) \times \overrightarrow{\boldsymbol{u}}_{i}^{k} \\
\boldsymbol{I}_{i i}=\sum_{i=1}^{N} m_{i}\left(\vec{r}_{i}^{k}-\vec{r}_{i i}^{k}\right)^{2} \\
\vec{r}_{i i}^{k}=\frac{1}{N} \sum_{i=1}^{N} \vec{r}_{i}^{k}
\end{array}\right.
$$

where $\vec{u}_{i}, \vec{r}_{i}, m_{i}$ are the velocity, position and mass of the moving particle, respectively, $\vec{u}_{i i}, \vec{\omega}_{i i}$ are the translation velocity and angular velocity of the solid body, respectively, and $I_{i i}, \vec{r}_{i i}$ are the moment of inertia and the center of gravity of the solid body, $\mathrm{N}$ is the number of moving particles in one solid body, $k$ is the number of time steps in the DEM calculation, $\vec{u}_{i}, \vec{r}_{i}$ are the initial velocity and position of moving particles, which are firstly provided by the MPS calculation.

After getting the initial velocity and position of the solid body, each solid body is regarded as one element in DEM calculation. When the distance between two solid bodies is less than the sum of their diameters, the two solid bodies are considered to be contact. The equations of motion by Newton's second law for each body are established as follows:

$$
\begin{aligned}
& \vec{a}_{i i}^{k}=\partial \vec{u}_{i i}^{k} / \partial t=\vec{F}_{c o l}^{k} / m_{i i} \\
& \vec{\alpha}_{i i}^{k}=\partial \vec{\omega}_{i i}^{k} / \partial t=\vec{T}_{c o l}^{k} / I_{i i}
\end{aligned}
$$

where $\vec{a}_{i i}^{k}, \vec{\alpha}_{i i}^{k}$ are acceleration and angular acceleration, respectively, $\vec{F}_{c o l}^{k}$ and $\vec{T}_{c o l}^{k}$ are the joint force and moment act on the solid body, $\mathrm{k}$ is the number of time steps in the DEM calculation. And the velocity, angular velocity and the position, rotation angle of the 
solid body in the next time step are:

$$
\begin{aligned}
& \vec{u}_{i i}^{k+1}=\vec{u}_{i i}^{k}+\vec{a}_{i i}^{k} \cdot \Delta t_{D E M} \\
& \vec{\omega}_{i i}^{k+1}=\vec{\omega}_{i i}^{k}+\vec{\alpha}_{i i}^{k} \cdot \Delta t_{D E M} \\
& \vec{r}_{i i}^{k+1}=\vec{r}_{i i}^{k}+\vec{u}_{i i}^{k} \cdot \Delta t_{D E M} \\
& \vec{\theta}_{i i}^{k+1}=\vec{\theta}_{i i}^{k}+\vec{\omega}_{i i}^{k} \cdot \Delta t_{D E M}
\end{aligned}
$$

The solid body moves to a new position in next time step, the joint force and moment also update, then calculating the $\vec{F}_{c o l}^{k+1}, \vec{T}_{c o l}^{k+1}$, and repeat this process, the movement of each solid body will be obtained.

The model of collision between solid bodies is introduced in Fig. 1 to get the joint force and moment of the solid body:

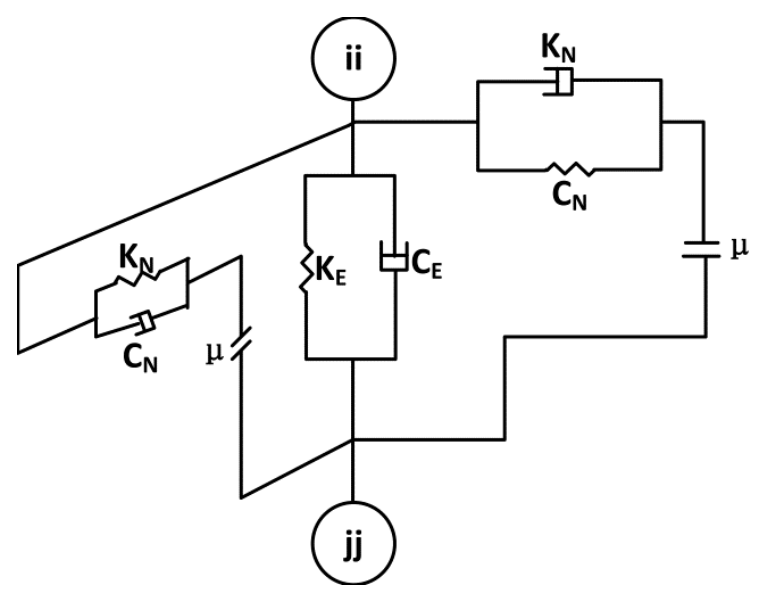

Fig. 1 The model of collision

As shown in Fig.1, two solid bodies interact by spring dampers and sliding graters, which stand for the elastic force, damping force and the maximum static friction force between solid bodies, respectively. In the normal direction of the collision, there are two spring dampers, while another two spring dampers and two sliding graters are set in each tangent direction. Besides that, there are one rotary damper and one rotary sliding graters circling the normal direction. If the joint force in the tangent direction is larger than the maximum static friction force between solid bodies, the sliding happens, the sliding graters work at this time, otherwise, the spring dampers work.

The local coordinate system is built with the gravity center of solid body $i i$ as the 
coordinate origin, the direction of normal as the $\mathrm{X}$ axis, the straight line parallels to the surface $x-y$ as the $Y$ axis, and the $Z$ axis is defined by right-hand screw rule. The schematic of the local coordinate system is shown in Fig.2. The relationship of physical quantities between local coordinate system and global coordinate system is shown as equation (18).

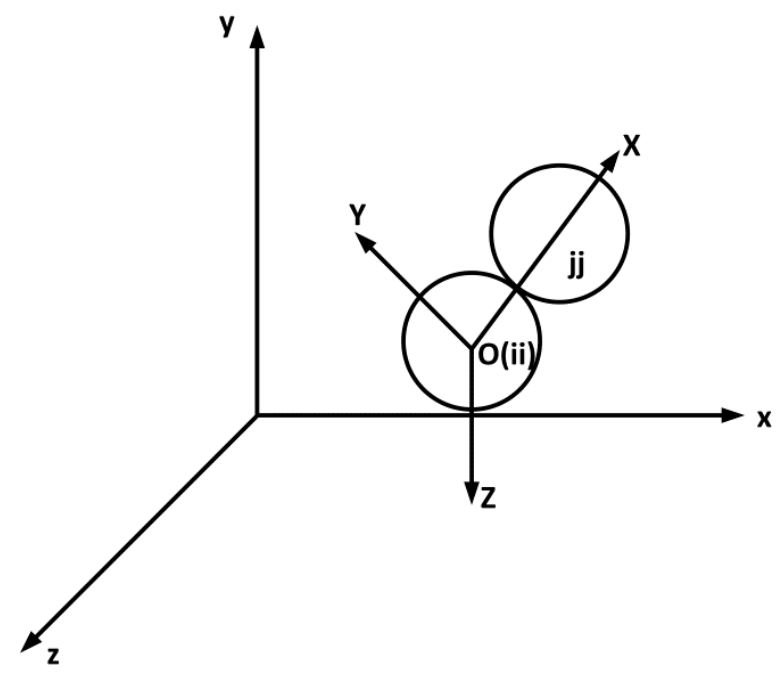

Fig.2 Schematic of the local coordinate system

$$
\left\{\begin{array}{l}
X \\
Y \\
Z
\end{array}\right\}=\left[M_{i i}\right]\left\{\begin{array}{l}
x \\
y \\
z
\end{array}\right\} \text { or }\left\{\begin{array}{l}
x \\
y \\
z
\end{array}\right\}=\left[M_{i i}\right]^{-1}\left\{\begin{array}{l}
X \\
Y \\
Z
\end{array}\right\}
$$

where $M_{i i}$ is the transition matrix between local coordinate system and global coordinate system. It is calculated as follows:

$$
M_{i i}=\left[\begin{array}{ccc}
l_{x} & m_{y} & n_{z} \\
-m_{y} / \sqrt{l_{x}^{2}+m_{y}^{2}} & l_{x} / \sqrt{l_{x}^{2}+m_{y}^{2}} & 0 \\
-l_{x} n_{z} / \sqrt{l_{x}^{2}+m_{y}^{2}} & -m_{y} n_{z} / \sqrt{l_{x}^{2}+m_{y}^{2}} & \sqrt{l_{x}^{2}+m_{y}^{2}}
\end{array}\right]
$$

where $l_{x}, m_{y}, n_{z}$ are the direction cosines of the $\mathrm{X}$ axis in the global coordinate system:

$$
\begin{aligned}
& l_{x}=\left(x_{j j}-x_{i i}\right) / D_{i j} \\
& m_{y}=\left(y_{j j}-y_{i i}\right) / D_{i j} \\
& n_{z}=\left(z_{j j}-z_{i i}\right) / D_{i j}
\end{aligned}
$$


where $x, y, z$ are the position of the gravity center of solid body in global coordinate system, $D_{i j}$ is the distance between two solid bodies.

In one time step of DEM calculation, the increment of the displacement and rotation angle of solid body $i i$ or $j j$ are:

$$
\begin{aligned}
\Delta \vec{r}_{i i(j)} & =\vec{u}_{i i(j j)} \cdot \Delta t_{D E M} \\
\Delta \vec{\theta}_{i i(j j)} & =\vec{\omega}_{i i(j j)} \cdot \Delta t_{D E M}
\end{aligned}
$$

According to equations (18), (23) and (24), the relative displacement and rotation angle between solid body $i i$ and $j j$ can be described as follows:

$$
\begin{aligned}
\left\{\begin{array}{l}
\Delta X_{i i, j j} \\
\Delta Y_{i i, j j} \\
\Delta Z_{i i, j j}
\end{array}\right\}= & {\left[M_{i i}\right]\left\{\begin{array}{l}
u_{i i}-u_{j j} \\
v_{i i}-v_{j j} \\
w_{i i}-w_{j j}
\end{array}\right\} \cdot \Delta t_{D E M}+\left\{\begin{array}{cc}
0 & 0 \\
\Delta \theta_{Z, i i} & \Delta \theta_{Z, j j} \\
-\Delta \theta_{Y, i i} & -\Delta \theta_{Y, i i}
\end{array}\right\}\left\{\begin{array}{l}
r_{i i} \\
r_{j j}
\end{array}\right\} } \\
& \left\{\begin{array}{l}
\Delta \theta_{X, i i, j j} \\
\Delta \theta_{Y, i i, j j} \\
\Delta \theta_{Z, i i, j j}
\end{array}\right\}=\left[M_{i i}\right]\left\{\begin{array}{l}
\omega_{x, i i}-\omega_{x, j j} \\
\omega_{y, i i}-\omega_{y, j j} \\
\omega_{z, i i}-\omega_{z, j j}
\end{array}\right\} \cdot \Delta t_{D E M}
\end{aligned}
$$

At time t, the elastic force and damping force in normal direction (Gotoh, $\mathrm{H}$. and Sakai, T., 1997) are modeled as follows:

$$
\left\{\begin{array}{l}
F_{X, j j \rightarrow i i}(t)=e_{n}(t)+d_{n}(t) \\
e_{n}(t)=e_{n}\left(t-\Delta t_{D E M}\right)+k_{E} \Delta X_{i i, j j} \\
d_{n}(t)=c_{E} \frac{\Delta X_{i i, j j}}{\Delta t_{D E M}}
\end{array}\right.
$$

The elastic force and damping force in tangent direction (Gotoh, H. and Sakai, T., 1997) are formulated in the same form :

$$
\left\{\begin{array}{l}
F_{Y(Z), j j \rightarrow i i}(t)=e_{s}(t)+d_{s}(t) \\
e_{s}(t)=e_{s}\left(t-\Delta t_{D E M}\right)+k_{N} \Delta Y(Z)_{i i, j j} \\
d_{s}(t)=c_{N} \frac{\Delta Y(Z)_{i i, j j}}{\Delta t_{D E M}}
\end{array}\right.
$$


In equations (27) and (28), $F_{X}, F_{Y(Z)}$ are the joint force in normal and tangent direction respectively, $e, d$ are the elastic force and damping force respectively, the subscript $n$, $s$ are represent normal direction and tangent direction, $k_{E}, c_{E}$ are the elastic coefficient and damping coefficient in normal direction and $k_{N}, c_{N}$ are those in tangent direction, respectively. In the local coordinate system, both the direction of the $\mathrm{Y}$ axis and $\mathrm{Z}$ axis belong to the direction of tangent, the force in these two direction are calculated in the same way, and all the calculation parameters except the force direction are also the same.

The movement of solid body in the tangent direction is influenced by the maximum static friction force between solid bodies, so the process is not continuous, the force in tangent direction should be corrected by static friction force. If $\sqrt{F_{Y, j j \rightarrow i i}^{2}(t)+F_{Z, j j \rightarrow i i}^{2}(t)}>\left|F_{X, j j \rightarrow i i}(t)\right| \cdot \mu$, the relative sliding happens between two solid bodies, the force in tangent direction is modified by static friction force as follows:

$$
F_{Y(Z), j j \rightarrow i i}(t)=\left|F_{X, j j \rightarrow i i}(t)\right| \cdot \mu \cdot F_{Y(Z), j j \rightarrow i i}(t) / \sqrt{F_{Y, j j \rightarrow i i}^{2}(t)+F_{Z, j j \rightarrow i i}^{2}(t)}
$$

It is remarkable that if $e_{n}(t) \leq 0$, the joint force in normal and tangent direction should be both modified to be zero, even if two solid bodies are very closed.

Similarly, in one time step of DEM calculation, the elastic increment and the damping component of the rotary moment are:

$$
\begin{gathered}
\Delta T_{X e, j j \rightarrow i i}=k_{R} \Delta \theta_{X, i i, j j} \\
T_{d, j j \rightarrow i i}=c_{R} \Delta \theta_{X, i i, j j} / \Delta t_{D E M}
\end{gathered}
$$

where $k_{R}, c_{R}$ are the rotary elastic coefficient and rotary damping coefficient, respectively, $\Delta T_{X e}$ is the elastic increment of rotary moment circling $\mathrm{X}$ axis, $T_{d}$ is the damping component of the rotary moment.

The coefficients in the DEM calculation can be formulated as follows (Gladwell G M L., 1980): 


$$
\begin{gathered}
k_{E}=\frac{8 r}{3\left(\theta_{i i}+\theta_{j j}\right)} \\
k_{N}=\frac{8 r}{\frac{2-v_{i i}}{G_{i i}}+\frac{2-v_{j j}}{G_{j j}}} \\
k_{R}=\frac{16 r^{3}}{3} \frac{G_{i i} G_{j j}}{G_{i i}+G_{j j}}
\end{gathered}
$$

where

$$
\begin{gathered}
r=\sqrt[3]{3 F_{X} R_{i i} R_{j j}\left(\theta_{i i}+\theta_{j j}\right) / 8\left(R_{i i}+R_{j j}\right)} \\
\theta=2\left(1-v^{2}\right) / E \\
G=E / 2(1-v)
\end{gathered}
$$

$r$ is the contact radius between two solid bodies, $E, R, v$ are the elastic modulus, radius of solid body and the Poisson ratio. But these parameters of material are hard to be confirmed and considering the computational efficiency, these equations are not adopted in FOCUS code, the coefficients of DEM calculation are set artificially instead.

According to equations (30) and (31), the elastic component of the rotary moment and the joint moment circling $\mathrm{X}$ axis are calculated as follows:

$$
\begin{gathered}
T_{X e, j j \rightarrow i i}(t)=T_{X e, j j \rightarrow i i}\left(t-\Delta t_{D E M}\right)+\Delta T_{X e, j j \rightarrow i i}(t) \\
T_{X, j j \rightarrow i i}(t)=T_{X e, j j \rightarrow i i}(t)+T_{d, j j \rightarrow i i}(t)
\end{gathered}
$$

If $\left|T_{X, j j \rightarrow i i}(t)\right|>\left|F_{X, j j \rightarrow i i}(t)\right| \cdot \mu \cdot r$, the moment also should be modified by static friction force as follows:

$$
T_{X, j j \rightarrow i i}(t)=\left|F_{X, j j \rightarrow i i}(t)\right| \cdot \mu \cdot r \cdot \operatorname{sign}\left(T_{X, j j \rightarrow i i}(t)\right)
$$

where $\operatorname{sign}\left(T_{X, j j \rightarrow i i}(t)\right)$ represents the sign of $T_{X, j j \rightarrow i i}(t)$.

After calculating the joint force and moment in local coordinate system, the corresponding physical quantities should be transformed into global coordinate system by equations as follows: 


$$
\begin{gathered}
\left\{\begin{array}{c}
F_{x, j j \rightarrow i i}(t) \\
F_{y, j j \rightarrow i i}(t) \\
F_{z, j j \rightarrow i i}(t)
\end{array}\right\}=\sum\left[M_{i i}\right]^{-1}\left\{\begin{array}{c}
F_{X, j j \rightarrow i i}(t) \\
F_{Y, j j \rightarrow i i}(t) \\
F_{Z, j j \rightarrow i i}(t)
\end{array}\right\} \\
\left\{\begin{array}{c}
T_{x, j j \rightarrow i i}(t) \\
T_{y, j j \rightarrow i i}(t) \\
T_{z, j j \rightarrow i i}(t)
\end{array}\right\}=\sum\left[M_{i i}\right]^{-1}\left\{\begin{array}{c}
T_{X, j j \rightarrow i i}(t) \\
0 \\
0
\end{array}\right\}+r_{i i} \sum\left[M_{i i}\right]^{-1}\left\{\begin{array}{c}
0 \\
F_{Y, j j \rightarrow i i}(t) \\
F_{Z, j j \rightarrow i i}(t)
\end{array}\right\}
\end{gathered}
$$

where $\sum$ is to sum all the force and moment that act on $i i$ by all the solid bodies which are contact with $i i$. The acceleration and angular acceleration are calculated by equations (12) and (13), and the velocity and position of solid bodies in the next time step are updated by equations (14) to (17). After getting the velocity and position of solid bodies, the velocity and position of the moving particle which form the solid body is calculated as follows:

$$
\begin{gathered}
\vec{u}_{i}^{k+1}=\vec{u}_{i i}^{k+1}+\vec{\omega}_{i i}^{k+1}\left(\vec{r}_{i}^{k}-\vec{r}_{i i}^{k}\right) \\
\vec{r}_{i}^{k+1}=\vec{r}_{i}^{k}+\vec{u}_{i}^{k+1} \cdot \Delta t_{D E M}
\end{gathered}
$$

\subsection{Coupling the DEM model with the MPS method}

The MPS method is applied to simulate the flow of fluid, while the DEM model can solve the movement of solid bodies. In this study, these two methods are coupled to simulate the fluid-solid mixture flow. The interaction force between fluid and solid bodies can be modeled as follows (Koshizuka, S., Nobe, A. et al., 1998):

$$
\vec{f}_{l s}=-(\nabla p)_{l s}+\nabla(\mu \cdot \nabla \vec{u})_{l s}
$$

where the first term on the right represents the pressure force between fluid and solid, the second term on the right is the interaction that caused by the viscosity of the fluid. The equation (45) can be solved by the particle interaction models in the MPS method, where the pressure force can be handled by equation (3) and viscosity force can be solved by equation (5).

The interaction between fluid and solid phase is one of the key steps in this coupling algorithm. After solving this problem, the way of coupling the DEM model with the MPS 
method is clear and described as follows.

First, all moving particles including those which form the solid bodies are considered as fluid particles, and all particles are calculated by the MPS method as described in Section 2.1. After the MPS calculation, the equation (45) has been solved and the interaction between fluid particles and solid particles has been considered. Each particle has the initial velocity calculated by the MPS method at this time. Then the translation velocity and angular velocity of solid bodies is initialized using equation (11) .

Thereafter, judging the contact between solid bodies, if collision happens, the collision effect is simulated by the DEM model as introduced in Section 2.2. The increases of the distance $\Delta X, \Delta Y, \Delta Z$ are updated by the translation velocity and angular velocity of solid bodies in each time step according to equation (25). The joint force and moment are calculated by equations (28) and (29).

It is remarkable that the time step of the DEM calculation is much smaller than that in the MPS calculation, the $\Delta t_{D E M}$ is about three order of magnitude smaller than the $\Delta t_{M P S}$. If the $\Delta t_{M P S}$ is used to be the time step of the whole process, the calculation result will not converge when calculate the collision between solid bodies. On the other hand, if the $\Delta t_{D E M}$ is used to be the time step of the whole process, the calculation will carry out very slowly. So a method of multiple time-step using inner iteration is introduced to cope this problem.

The basic idea of the multiple time-step method is that, in the MPS calculation, the $\Delta t_{M P S}$ is used, when the collision happens in one MPS time step, the DEM calculation carries out by $\Delta t_{D E M}$ as follows:

$$
\begin{aligned}
& \vec{u}_{i i}^{n, k+1}=\vec{u}_{i i}^{n, k}+\Delta t_{D E M} \frac{\vec{F}_{c o l, i i}^{n, k}}{m_{i i}} \\
& \vec{\omega}_{i i}^{n, k+1}=\vec{\omega}_{i i}^{n, k}+\Delta t_{D E M} \frac{\vec{T}_{c o l, i i}^{n, k}}{I_{i i}}
\end{aligned}
$$

where $\mathrm{k}$ is the number of the time steps in the DEM calculation and $\mathrm{n}$ is the number of the 
time steps in the MPS calculation, the initial values of $\vec{u}_{i i}^{n, 0}$ and $\vec{\omega}_{i i}^{n, 0}$ are calculated by the MPS method and modified by equation (7).

In each time step of DEM calculation, the translation velocity and angular velocity of each solid body are calculated by equations (27), (28), (46), (47), and position and rotation angle are updated as follows:

$$
\begin{aligned}
& \vec{r}_{i i}^{n, k+1}=\vec{r}_{i i}^{n, k}+\vec{u}_{i i}^{n, k+1} \cdot \Delta t_{D E M} \\
& \vec{\theta}_{i i}^{n, k+1}=\vec{\theta}_{i i}^{n, k}+\vec{\omega}_{i i}^{n, k+1} \cdot \Delta t_{D E M}
\end{aligned}
$$

After getting the position and velocity of each solid body, the position and velocity of moving particles which form the solid bodies are modified as follows:

$$
\begin{gathered}
\vec{u}_{i}^{n, k+1}=\vec{u}_{i i}^{n, k+1}+\vec{\omega}_{i i}^{n, k+1}\left(\vec{r}_{i}^{n, k}-\vec{r}_{i i}^{n, k}\right) \\
\vec{r}_{i}^{n, k+1}=\vec{r}_{i}^{n, k}+\vec{u}_{i}^{n, k+1} \cdot \Delta t_{D E M}
\end{gathered}
$$

Finally, the inner iteration carries out till $k+1=\frac{\Delta t_{M P S}}{\Delta t_{D E M}}$, the $\mathrm{k}+1$ inner iterations of DEM calculation has finished, the position and velocity of moving particles at this time in DEM calculation is adopted to be the position and velocity in the next MPS time step:

$$
\begin{aligned}
& \vec{u}_{i}^{n+1}=\vec{u}_{i}^{n, k+1} \\
& \vec{r}_{i}^{n+1}=\vec{r}_{i}^{n, k+1}
\end{aligned}
$$

\section{Compilation of FOCUS code}

\subsection{Compilation of DEM model}

In this study, a DEM model is developed based on a MPS code using $\mathrm{C} \# / \mathrm{C}_{++}$ language. The MPS method is a meshless numerical method which divided the continuum mechanics into moving particles, the solid bodies in the DEM calculation can also be represented by moving particles, so the pre-processing and post-processing parts of MPS can be directly adopted in DEM model, it is very convenient for the compilation.

There are always contacts and separations between solid bodies in every time step of DEM calculation, so the position of each solid body should be checked in every time step to judge if the collision happens. If every solid body in the whole calculation domain is 
searched in every time step, it will be a large computational amount and data amount. In order to improve the computational efficiency, the calculation domain is divided into many sub-domains which are called buckets in FOCUS code. In each bucket, one solid body just need to calculate the collision effects between those in the same bucket.

In DEM calculation, velocity, position and joint force of each solid body are updated after every time step. In FOCUS code, the initial velocity of each moving particle in DEM calculation is provided by the MPS calculation, if the MPS calculation is not involved in the simulation, the initial velocity is provided by gravity.

It is remarkable that the elastic force in the DEM model is formulated as the incremental form, that means every elastic force between solid bodies in this time step should be stored and used as the initial value in the next time step. If there are too many solid bodies in the calculation, the two-dimensional arrays used to store the elastic force will take up large memory, it will influence the efficiency of calculation.

The flowchart of the DEM calculation is shown as Fig.3. 


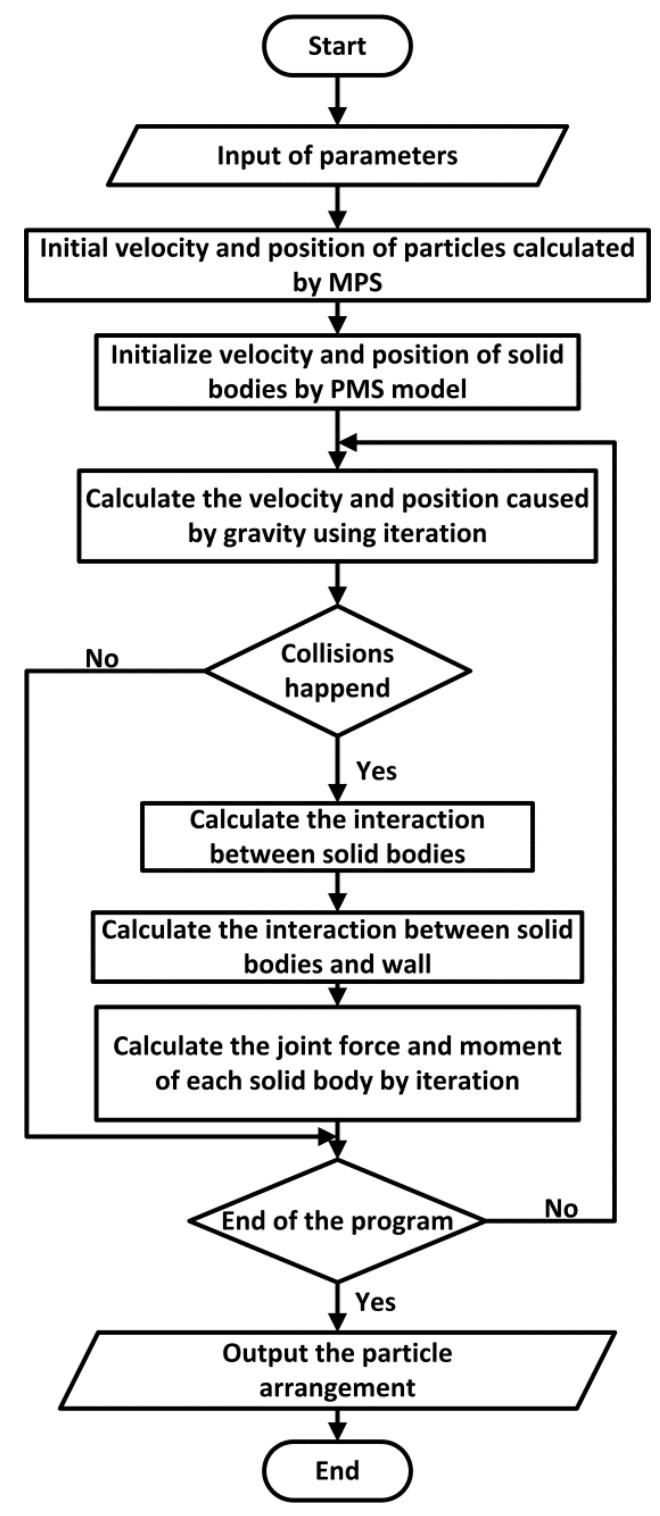

Fig.3 Flowchart of the DEM calculation

\subsection{Compilation of FOCUS code}

The FOCUS code is developed by coupling the DEM model with the MPS method, it can be adopted to simulate the fluid-solid mixture flow. The calculation process is summarized as follows:

(1) Set the initial particle arrangement according to the simulation case, including position $\vec{r}_{i}^{0}$, velocity $\vec{u}_{i}^{0}$ and pressure $p_{i}^{0}$ of each particle;

(2) Consider all the particles as fluid particles, and calculate the velocity and position of each particle, the initial velocity $\vec{u}_{i}^{n, 0}$ an position $\vec{r}_{i}^{n, 0}$ of solid particles are obtained;

(3) Modify the velocity $\vec{u}_{i i}^{n, 0}$ and gravity center $\vec{r}_{i i}^{n, 0}$ by the PMS model; 
(4) Judgment of collisions: if $D_{i j}<r_{i i}+r_{j j}$, carry out the DEM calculation;

(5) After $\mathrm{k}+1$ inner iterations, the velocity $\vec{u}_{i i}^{n, k+1}$ and gravity center $\vec{r}_{i i}^{n, k+1}$ of solid bodies are obtained;

(6) Calculate the velocity $\vec{u}_{i}^{n, k+1}$ and position $\vec{r}_{i}^{n, k+1}$ of solid particles by the velocity $\vec{u}_{i i}^{n, k+1}$ and gravity center $\vec{r}_{i i}^{n, k+1}$ of solid bodies;

(7) Use the velocity $\vec{u}_{i}^{n, k+1}$ and position $\vec{r}_{i}^{n, k+1}$ as the velocity $\vec{u}_{i}^{n+1}$ and position $\vec{r}_{i}^{n+1}$ in next time step of the MPS calculation;

(8) Repeat the process above;

The flowchart of the coupling calculation is shown as Fig.4.

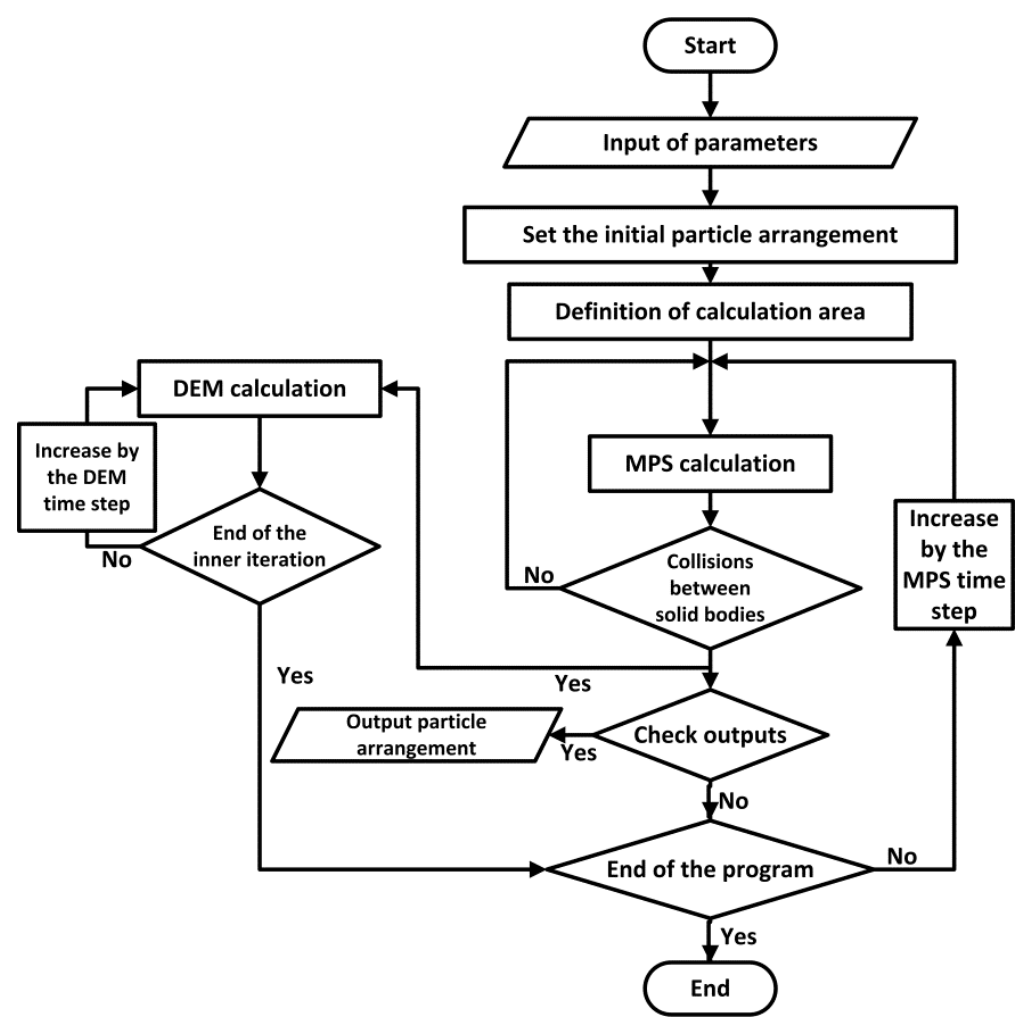

Fig.4 Flowchart of the FOCUS code

\section{Results and discussion}

\subsection{Validation of DEM model}

To verify the correctness and the reliability of the DEM model, a two-dimensional calculation example about the collapse of solid cylinder layers (Zhang, S., Morita, K., et al., 2009 ) is simulated. The reason why a two- dimensional calculation case is simulated is 
that both the direction of $Y$ axis and $Z$ axis in the local coordinate system belong to direction of tangent, the calculation parameters are all the same in these two directions, it means that if the two-dimensional calculation is verified to be correct, the calculation can be extended to the three-dimensional situations easily. What's more, the two-dimensional calculation is easier to be post-processed and has higher calculation efficiency, so the two-dimensional calculation example is finally chosen. The schematic of the collapse of solid cylinder layers is shown in Fig.5.

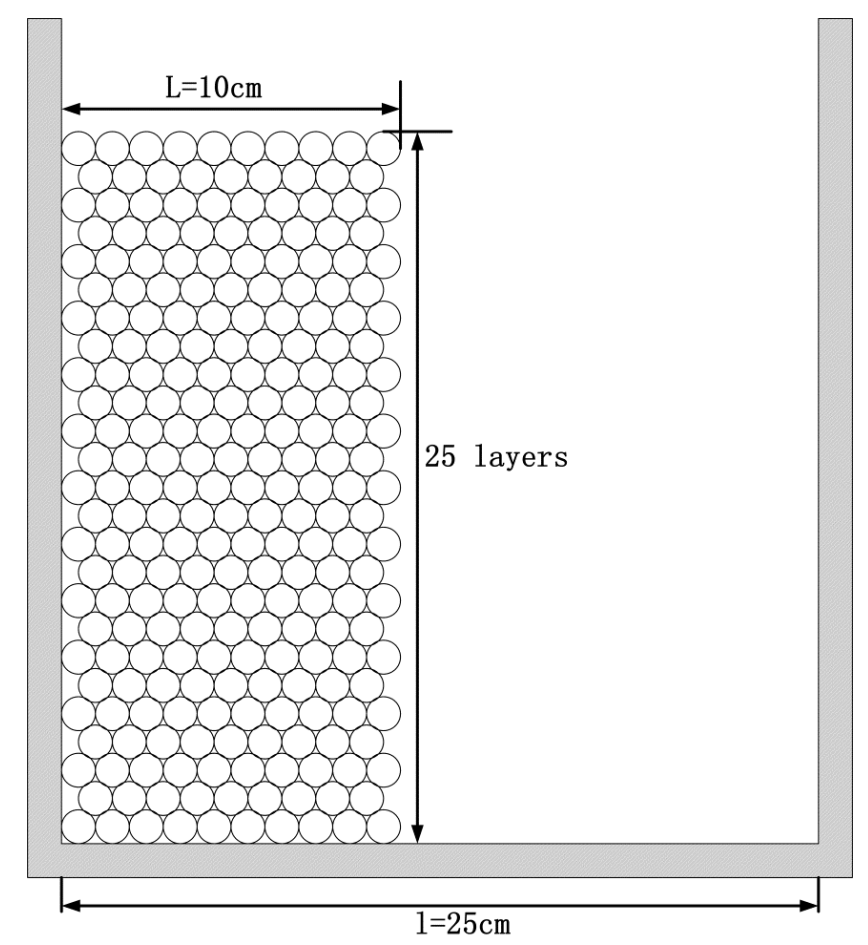

Fig.5 Schematic of the collapse of solid cylinder layers

As shown in Fig.4, the length of the container $l$ is $25 \mathrm{~cm}, 238$ cylinders whose diameter is $1 \mathrm{~cm}$ pile up at the left part of the container, the initial length between the right-most cylinder and the left wall $L$ is $10 \mathrm{~cm}$, and there are 25 layers of the cylinders. The initial particle arrangement for simulation is shown as Fig.6. In this simulation one solid body is represented by one single moving particle, and the MPS calculation is not involved, the initial velocity of each particle is provided by gravity. 


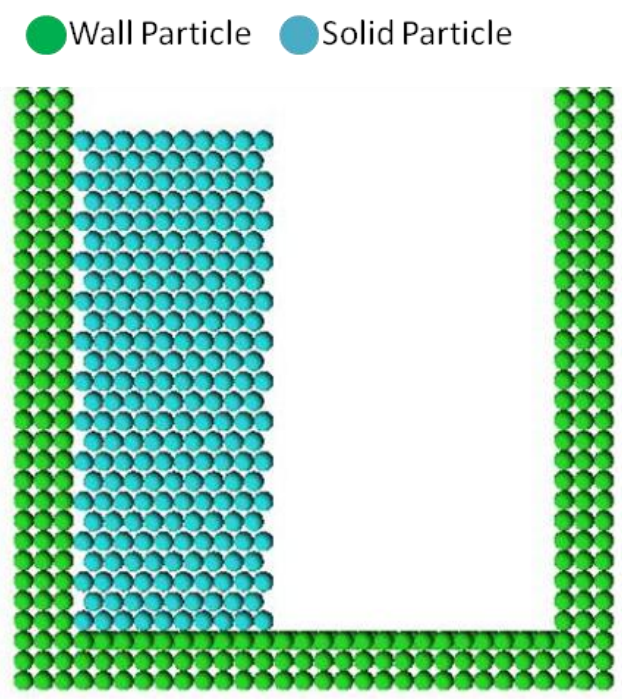

Fig.6 Initial particle arrangement for collapse of solid cylinder layers

The simulation result is correct or not, should be still validated by the experiments. In this study, an experiment about collapse of solid ball layers is carried out to validate the correctness of the simulation. The initial arrangement of the experiment is shown in Fig.7.

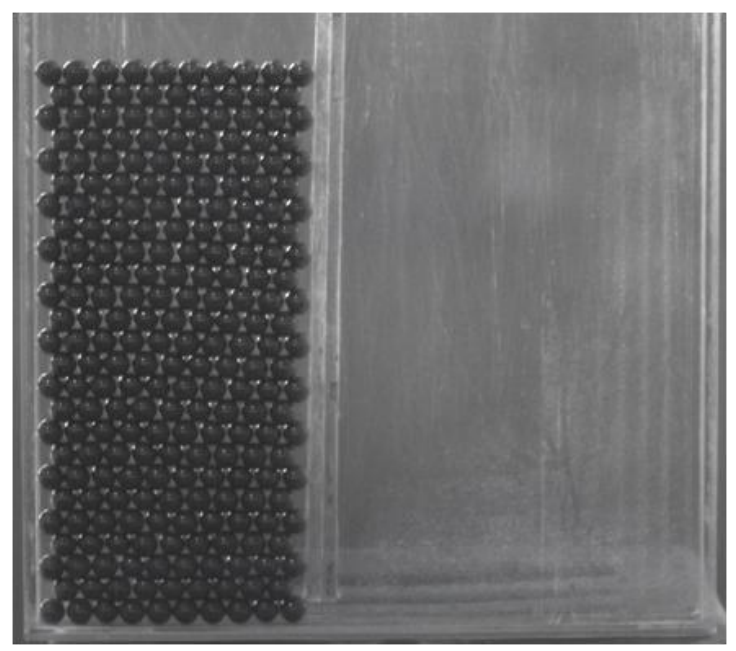

Fig.7 Initial experimental arrangement for collapse of solid ball layers

As shown in the figure, the experiment is designed in the same size of simulation, the $1 \mathrm{~cm}$ stainless steel ball is used in the experiment instead of the cylinders, the thickness of the container is precisely the diameter of the stainless steel ball to make sure the movement of balls in the direction of $z$ axis doesn't occur, so the experiment can be regarded as a two-dimensional experiment. On the right side of the ball layers, a baffle is set to prevent the movement of balls before experiment starts, then remove the baffle, the movement of balls is observed and recorded by the high-speed camera. 
The determination of each calculation parameter is the key point in the simulation, the parameters include elastic coefficient in normal direction $k_{E}$, damping coefficient in normal direction $c_{E}$, elastic coefficient in tangent direction $k_{N}$, damping coefficient in tangent direction $c_{N}$, rotary elastic coefficient $k_{R}$, rotary damping coefficient $c_{R}$, and maximum static friction coefficient $\mu$.

In these parameters, the elastic coefficient and the damping coefficient in each direction have an important impact on the result, due to the very small time step in the DEM calculation, as long as the coefficients in the calculation are at the same order of magnitude with the real value of each material, a convergent simulation result can be obtained. In this calculation, $k_{E}, k_{N}$ and $k_{R}$ are set as $10^{6} \mathrm{~N} / \mathrm{m}, 10^{5} \mathrm{~N} / \mathrm{m}$ and $100 \mathrm{~N} / \mathrm{m}$, respectively. $c_{E}, c_{N}$ and $c_{R}$ are set as $100 \mathrm{~N} / \mathrm{m}, 10 \mathrm{~N} / \mathrm{m}$ and $100 \mathrm{~N} / \mathrm{m}$, respectively, the time step of DEM calculation is set as $10^{-7} \mathrm{~s}$. In this simulation, the MPS calculation is not involved, so the time step of MPS calculation is not necessary, but in the later simulation about the fluid-solid mixture flow in this paper, the time step of MPS calculation should be set as $5 \times 10^{-5} \mathrm{~s}$.

The maximum static friction coefficient $\mu$ is another important factor in DEM calculation which can influence the sliding between solid cylinders. A larger value of $\mu$ will lead to a lager maximum static friction force which can prevent the movement of solid cylinders. However, if the value of $\mu$ is too small, the binding force between solid cylinders will not be enough, and the calculation result will distort. The maximum static friction coefficient of the stainless steal is between 0.1 and 0.3 , which varies with the different surface roughness. To determine the value of the maximum static friction coefficient, further research is done. Fig. 8 shows the calculation result at $0.4 \mathrm{~s}$ which $\mu=0.1$ and $\mu=0.3$ respectively, Fig. 9 shows the experimental result at $0.4 \mathrm{~s}$. 


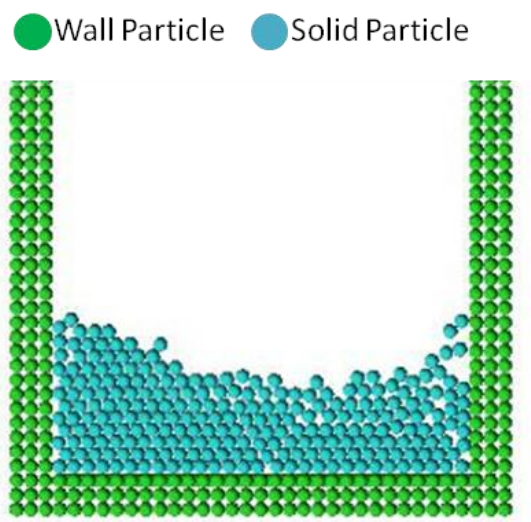

(a) $\mu=0.1$

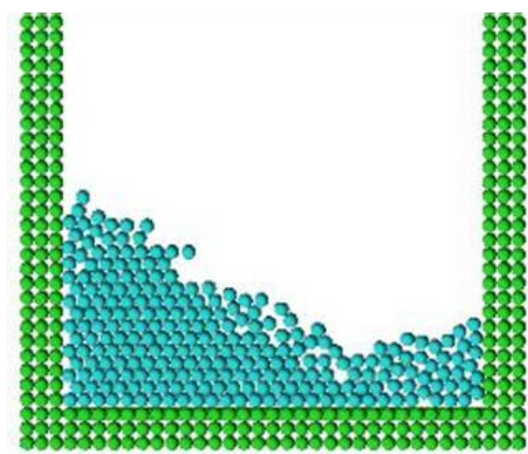

(b) $\mu=0.3$

Fig.8 Calculation results at $0.4 \mathrm{~s}$

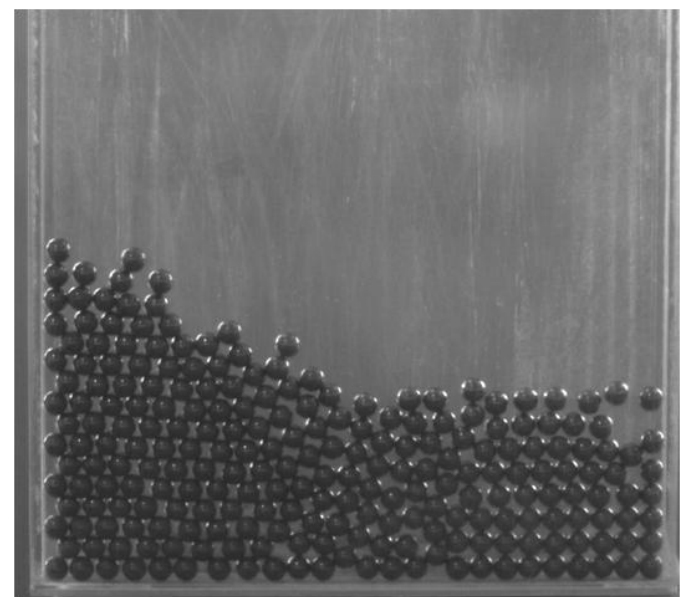

Fig.9 Experimental results at $0.4 \mathrm{~s}$

Comparing Fig. 8 and Fig.9, it can be concluded that when $\mu=0.1$, the movement of the cylinders is too fast and violent, this is because the maximum static friction force is too small between cylinders. On the other hand, when $\mu=0.3$, the maximum static friction force is so large that the movement of cylinders is prevented, so the valve of $\mu$ should be chosen between 0.1 and 0.3 . After further attempts, $\mu$ is finally set as 0.2 in this calculation. The density of the solid is set as $7930 \mathrm{~kg} / \mathrm{m}^{3}$. All the calculation parameters are summarized in Table.1.

Table.1 Parameters in DEM calculation

Parameters in DEM calculation

$k_{E}$ $10^{6} \mathrm{~N} / \mathrm{m}$ 


\begin{tabular}{cc}
\hline$c_{E}$ & $100 \mathrm{~N} / \mathrm{m}$ \\
$k_{N}$ & $10^{5} \mathrm{~N} / \mathrm{m}$ \\
$c_{N}$ & $10 \mathrm{~N} / \mathrm{m}$ \\
$k_{R}$ & $100 \mathrm{~N} / \mathrm{m}$ \\
$c_{R}$ & $10 \mathrm{~N} / \mathrm{m}$ \\
$\mu$ & 0.2 \\
Time step in DEM & $10^{-7} \mathrm{~s}$ \\
Time step in MPS (if necessary) & $5 \times 10^{-5} \mathrm{~s}$
\end{tabular}

The calculation results at $0.1 \mathrm{~s}, 0.2 \mathrm{~s}, 0.3 \mathrm{~s}$ and $0.4 \mathrm{~s}$ are shown in Fig.10, respectively. At $0.1 \mathrm{~s}$, the collapse of cylinders is just beginning, the cylinders in the upper-right corner start rolling down, those in the lower-left corner stay still, and between these two areas, there is a interface appears; At 0.2s, the cylinders in the lower-left corner still don't move, and the cylinders in the upper-right corner keep falling down; At 0.3s, the cylinders arrive at the left wall of container; At $0.4 \mathrm{~s}$, the process of collapse is over, and the height of the cylinder layers on the left part is larger than that on the right part.

As the comparison to the calculation results, the experiment results are also shown in Fig.10. According to the comparison, it can be concluded that the results of calculation are in good agreement with the experimental results. The simulation has successfully described the movement tendency of the cylinders, and the position of each cylinder in calculation is also fits well to experimental results at each time. The simulation also gives an accurate description about some individual cylinders as shown in the red ellipses in Fig.10. 


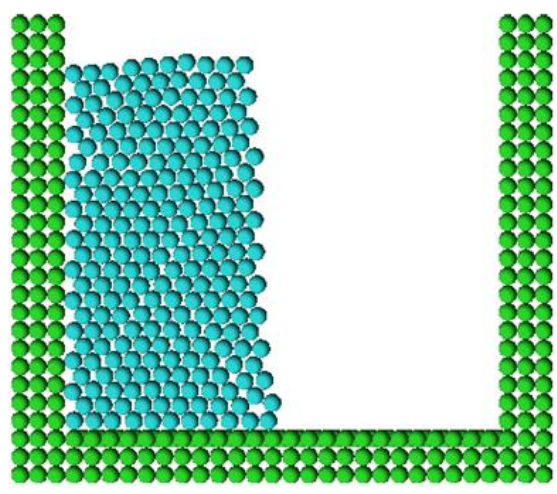

(a) $0.1 \mathrm{~s}$ in calculation

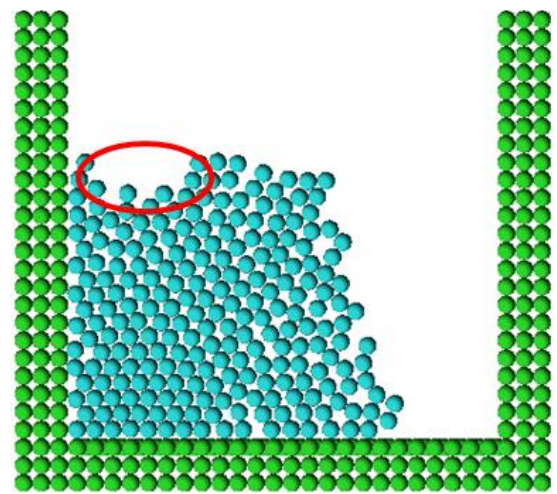

(c) $0.2 \mathrm{~s}$ in calculation

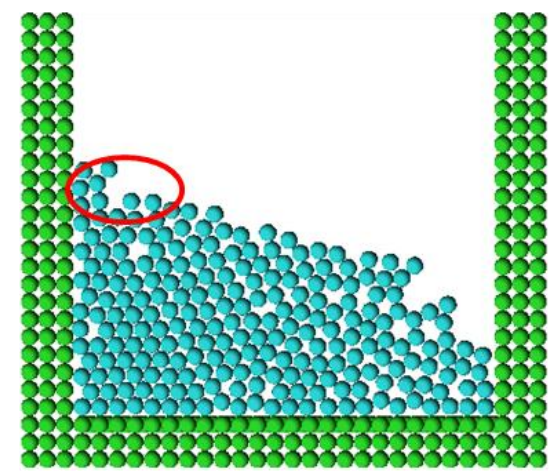

(e) $0.3 \mathrm{~s}$ in calculation

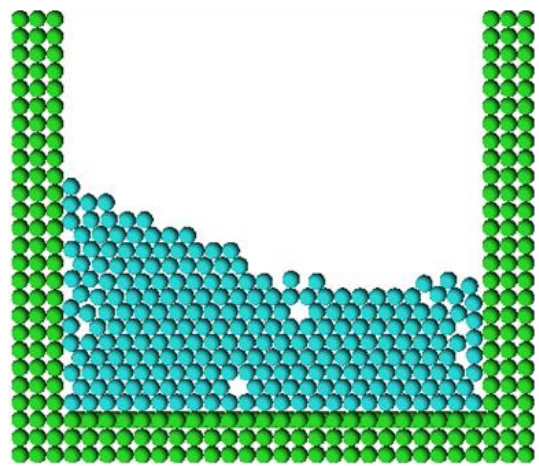

(g) $0.4 \mathrm{~s}$ in calculation

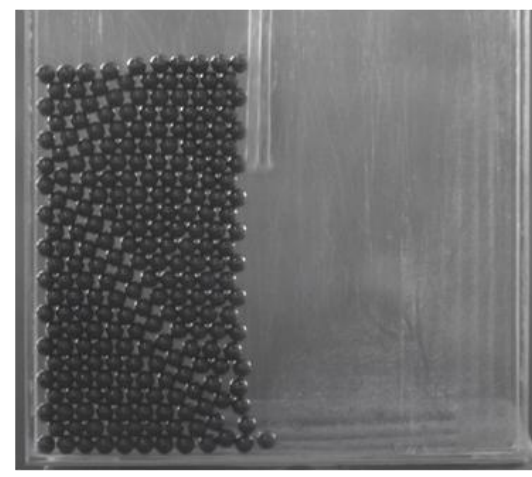

(b) $0.1 \mathrm{~s}$ in experiment

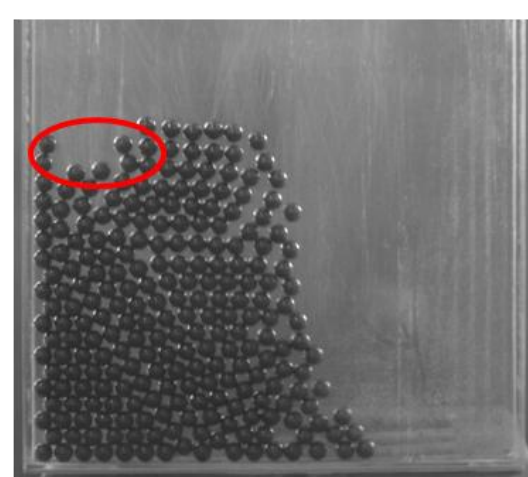

(d) $0.2 \mathrm{~s}$ in experiment

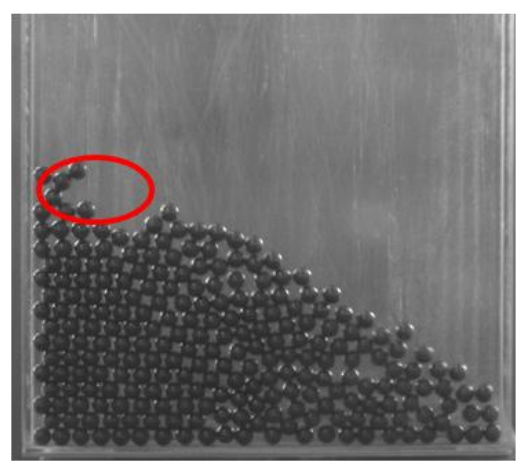

(f) $0.3 \mathrm{~s}$ in experiment

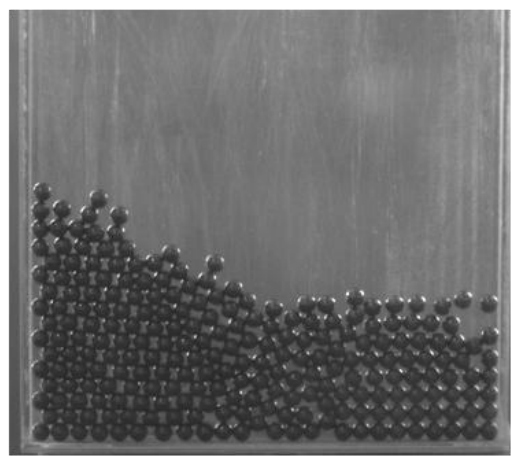

(h) $0.4 \mathrm{~s}$ in experiment 
Fig.10 Comparison between calculation results and experimental results of collapse of solid cylinder layers

To make the comparison between the calculation and the experiment more intuitive, the further processing of the calculation results and experimental data is carried out. Considering the relationship between horizontal displacement $\mathrm{X}$ and time $\mathrm{t}$ :

$$
X \sim g t^{2}
$$

where $\mathrm{X}$ is the horizontal displacement of the right-most cylinder when collapse happens. Thereafter, both side of equation (44) divided by the initial length between the right-most cylinder and the left wall $L_{1}$ :

$$
\frac{X}{L_{1}} \sim \frac{g t^{2}}{L_{1}}
$$

Then the equation (45) is rewritten as a quadratic function:

$$
\frac{X}{L_{1}} \sim\left(t \sqrt{\frac{g}{L_{1}}}\right)^{2}
$$

Finally, the relationship between the dimensionless vertical coordinate $X / L_{1}$ and the dimensionless horizontal coordinate $t \sqrt{g / L_{1}}$ is obtained, as shown in Fig.11.

In the same way, the relationship between vertical displacement $Y$ and time $t$ is shown in Fig.12. In Fig.12, Y is the vertical displacement of the cylinder at the upper-right corner of the layers, $\mathrm{H}$ is the initial height of the solid layers. 


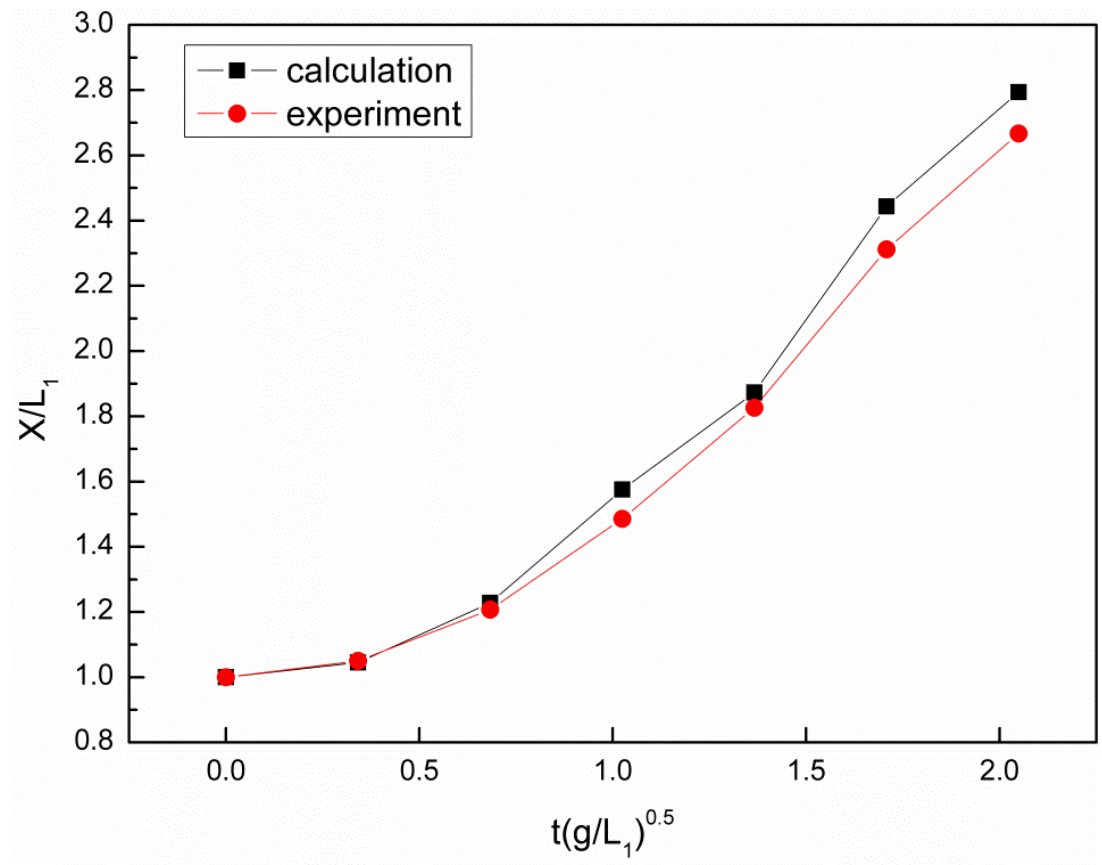

Fig.11 The relationship between the horizontal displacement of the right-most cylinder and time

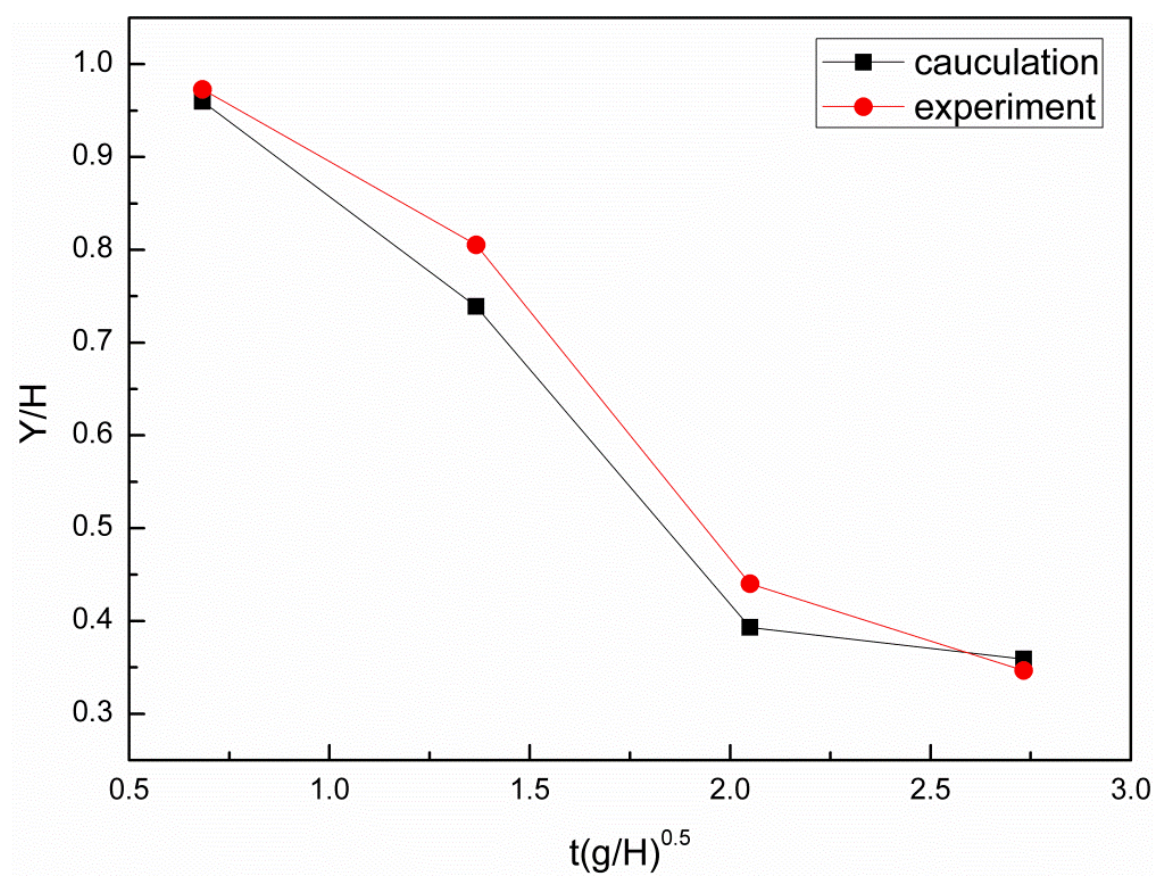

Fig.12 The relationship between the vertical displacement of the cylinder at the upper-right corner of the layers and time

In Fig.11 and Fig.12, the red lines and black lines are represent the calculation result and experimental data, respectively. It shows that the movement of cylinders in calculation and that in experiment is in good agreement with the average relative error less than $8.4 \%$. 
But the displacement in the calculation is a little larger than that in the experiment, it is because that there are front and rear panels of container which can act the friction force on solid bodies in experiment, and the baffle in experiment can also slow the movement of them, so the cylinders in calculation move faster than the experimental data. Overall, the program simulates the collapse of solid cylinder layers successfully, and the DEM model is validated to be correct by comparing the simulation results and the experimental data.

\subsection{Validation of fluid-solid mixture flow}

The simulation of fluid-solid mixture flow needs the coupling algorithm of DEM calculation and the MPS calculation, a two-dimensional calculation example about the breaking of a water dam involving solid cylinder layers ((Zhang, S., Morita, K., et al., 2009)) is used to verified the correctness of the coupling algorithm. The schematic of this calculation example is shown in Fig.13.

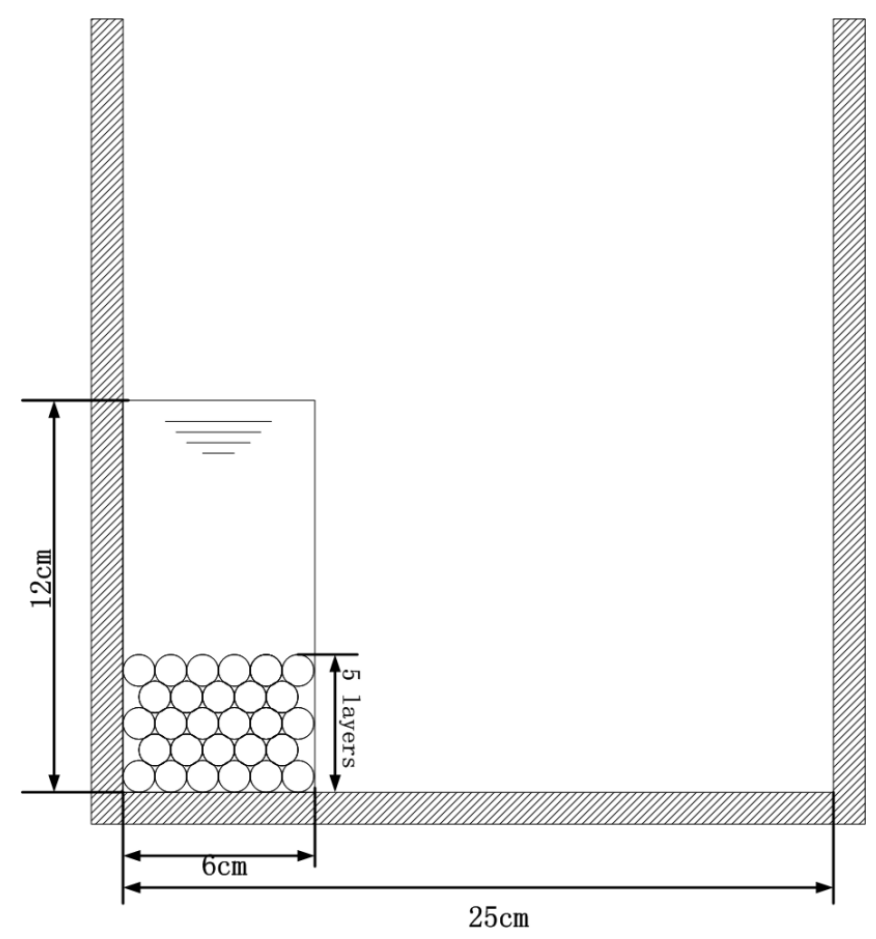

Fig.13 Schematic of the breaking of a water dam involving solid cylinder layers

As shown in Fig.13, size of the container is as the same as that shown in Fig.4. There are five layers piled in water at the left-lower corner of the container and the water level is $12 \mathrm{~cm}$ height. Twenty eight cylinders whose diameter is $1 \mathrm{~cm}$ are used in this calculation. The initial particle arrangement of this calculation example is shown in Fig.14. 


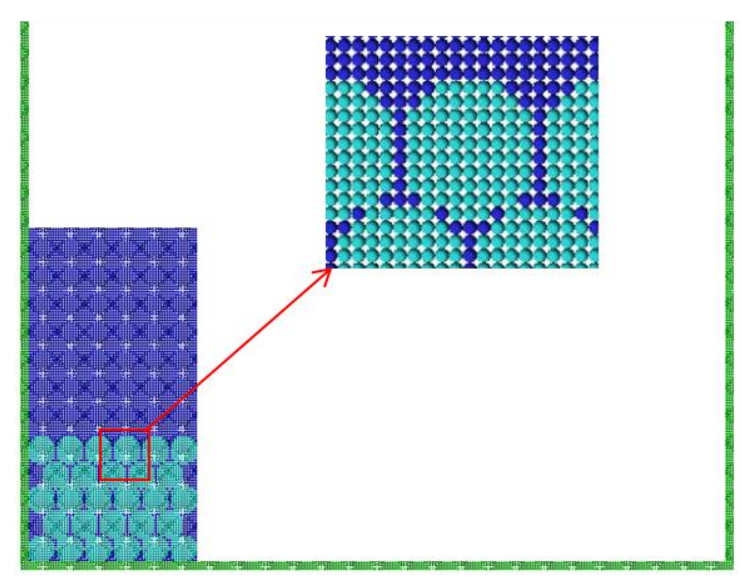

Fig.14 Initial particle arrangement of the breaking of a water dam involving solid cylinder layers

In this simulation, the size of particles is set as $1 \mathrm{~mm}$, and one cylinder consists of 69 moving particles as shown in Fig.14. The parameters include elastic coefficient, damping coefficient and maximum static friction coefficient in the DEM calculation are the same as those shown in Table.1, and the time steps in the MPS calculation and DEM calculation are set as $5 \times 10^{-5} \mathrm{~s}$ and $10^{-7} \mathrm{~s}$, respectively, the density of fluid and solid are set as $1000 \mathrm{~kg} / \mathrm{m}^{3}$ and $7930 \mathrm{~kg} / \mathrm{m}^{3}$. The calculation of the breaking of a water dam involving solid cylinder layers is an important case that verifies the program capability of simulation the fluid-solid mixture flow, the PMS model formulated in equation (11) and the interaction between fluid and solid adopted in equation (45) are both verified in this case.

Besides the simulation, an experiment about this case is carried out to validate the calculation results. As shown in Fig.15, the geometry dimension of the experimental equipment is the same as those shown in Fig.13, including the $1 \mathrm{~cm}$ diameter stainless steel balls, $12 \mathrm{~cm}$ in height of water level and the same size of the container which is made of plexiglass. What's more, there is a baffle with the sealant strip which is used to prevent the movement of the water dam and balls before experiment starts. The experimental results that recorded by the high-speed camera are shown in Fig.16. 


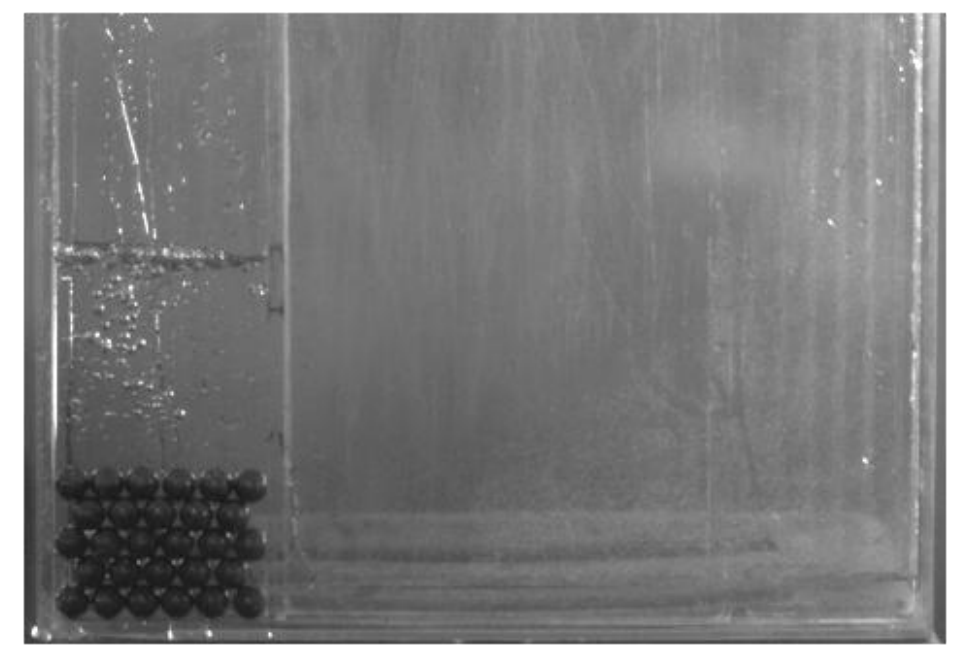

Fig.15 Initial experimental arrangement of the breaking of a water dam involving solid cylinder layers

The calculation results of this case are also shown in Fig.16. At $0.2 \mathrm{~s}$, the fluid has arrived at the right wall of the container, the shape of the water dam becomes a right triangle. The cylinders distribute in the water, the left-lower part of the cylinders which haven't moved yet become a shape of square, the middle part of the cylinders become a triangle shape, and the right-upper part of the cylinders are moving toward the right wall of container with the water wave. At $0.3 \mathrm{~s}$, the fluid-solid mixture flow keeps developing, the cylinders in the left-lower square also move with fluid, and the whole area of water become more gentle. The right-most cylinder is lifted by wave at this time. At $0.4 \mathrm{~s}$, the right-most cylinder falls down, and the right part of cylinders pile up with two layers at the bottom of the container.

The same times in experiment are selected as those in calculation, it shows the great agreement between calculation result and the experimental phenomena at each time. For example in Fig.16 (c) and Fig.16 (d), there are both three parts of solid bodies can be observed, including the left-lower square, the middle triangle and the right-lower ellipse. 
Wall Particle Solid Particle Pluid Particle

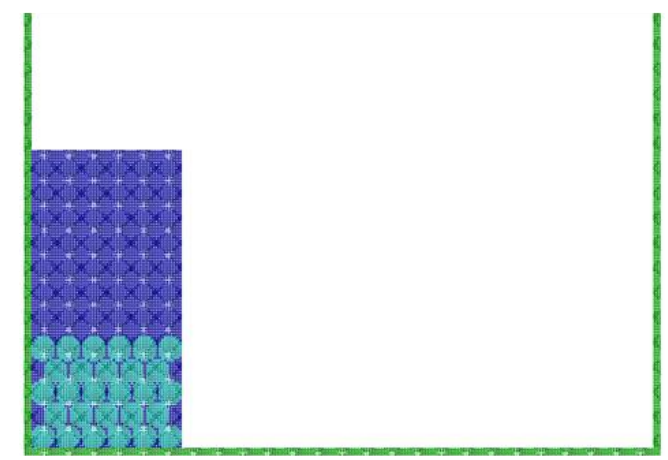

(a) 0.0 s in calculation

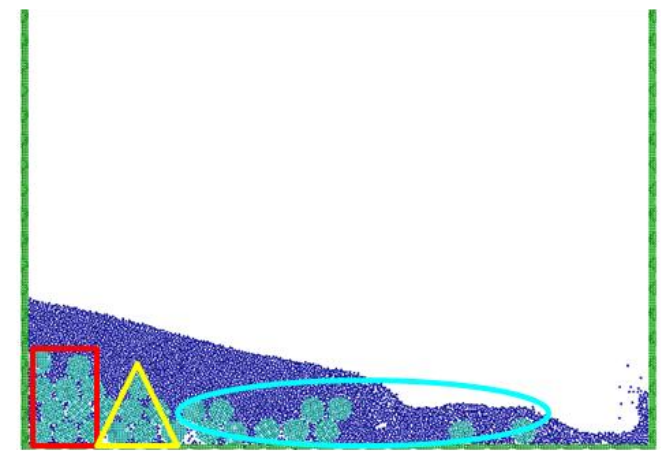

(c) $0.2 \mathrm{~s}$ in calculation

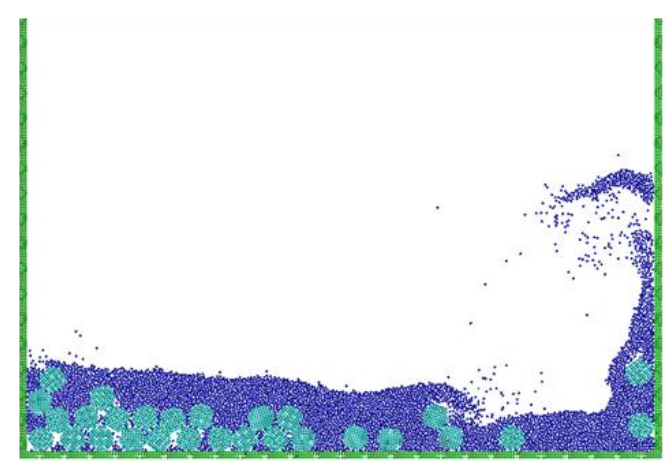

(e) $0.3 \mathrm{~s}$ in calculation

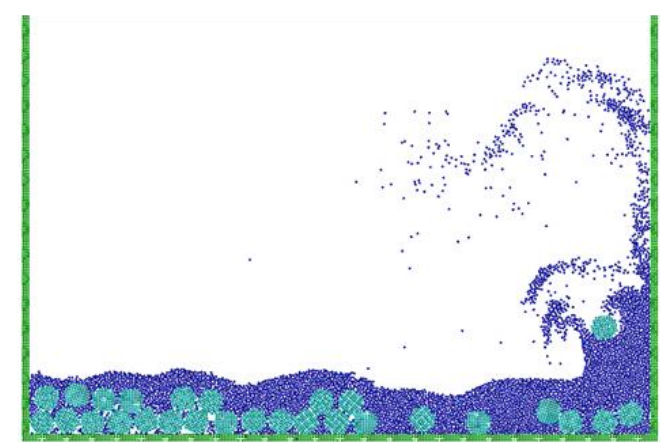

(g) $0.4 \mathrm{~s}$ in calculation

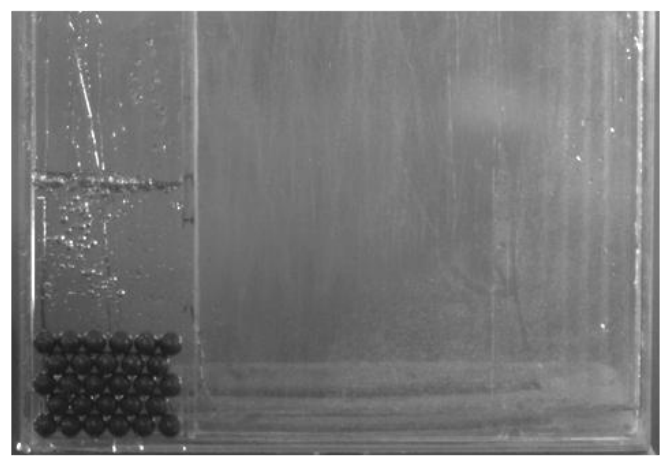

(b) 0.0 s in experiment

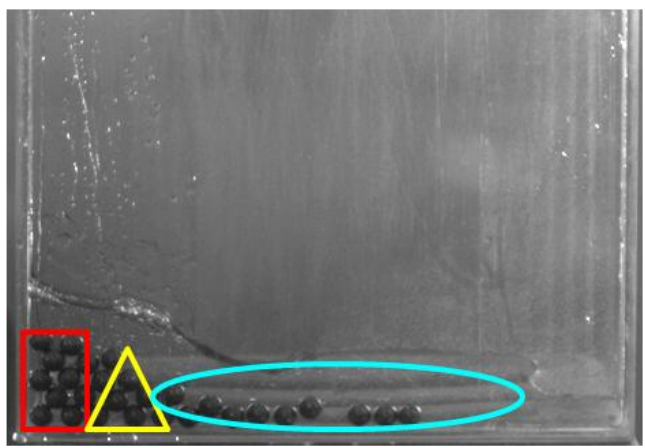

(d) $0.2 \mathrm{~s}$ in experiment

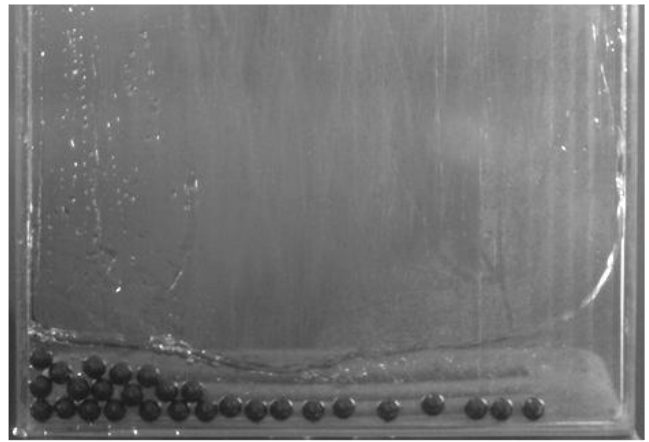

(f) $0.3 \mathrm{~s}$ in experiment

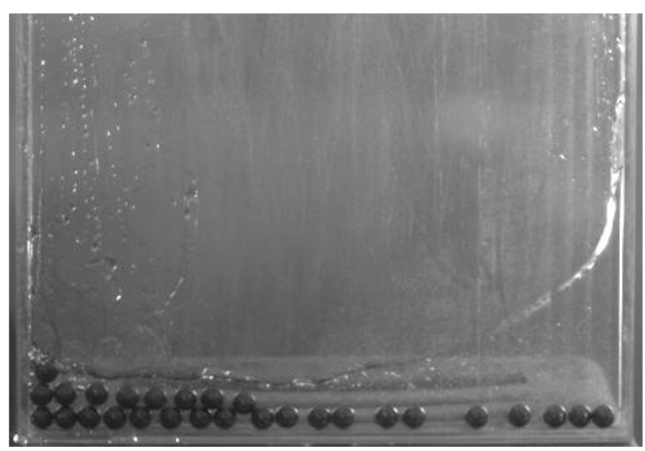

(h) $0.4 \mathrm{~s}$ in experiment

Fig.16 Comparison between calculation results and experimental results of 
the breaking of a water dam involving solid cylinder layers

It is remarkable that the solid bodies in the experiment move slower than those in the calculation, and the right-most solid body was lifted up by the water wave in calculation, but the same phenomenon is not observed in the experiment. That is because when the experiment starts, the baffle is removed to release the water dam and the solid layers, at the very time of drawing off the baffle, the water flows through the gap between the baffle and the bottom of the container immediately, but solid bodies do not move until the opening of the baffle lager than the diameter of the stainless steel ball. Thus in the experiment, the movement of the solid bodies is left behind by the fluid, but in the calculation , there is no influence of the baffle, the solid bodies move with the water dam at the same time. Finally, the right-most cylinder was lashed by the wave and lifted up. Considering the mass of one stainless steel ball is only 3 gram, the phenomenon is completely reasonable.

According to the data processing of Section 4.1, the quantitative comparisons between experiment and calculation are shown in Fig.17 and Fig.18. In Fig.17, $\bar{X}$ is the average horizontal position of solid bodies:

$$
\bar{X}=\frac{\sum x_{i i}}{N}
$$

where $x_{i i}$ is the horizontal position of each solid body, $\mathrm{N}$ is the number of solid bodies in this calculation example, $L_{2}$ is the initial length of the solid layers.

In Fig.18, $\bar{Y}$ is the average vertical position of solid bodies:

$$
\bar{Y}=\frac{\sum y_{i i}}{N}
$$

where $y_{i i}$ is the vertical position of each solid body, $\mathrm{H}$ is the initial height of the solid layers. 


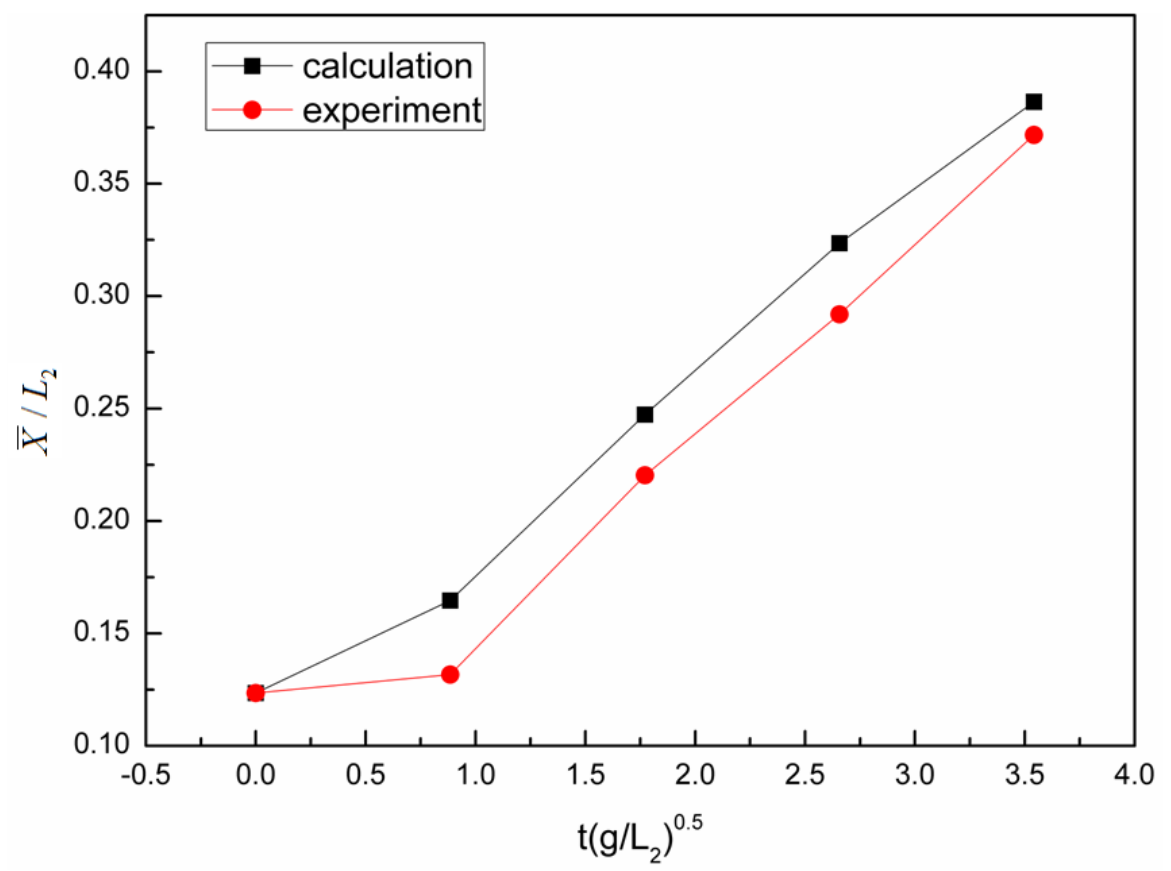

Fig.17 The relationship between the average horizontal position of solid bodies and time

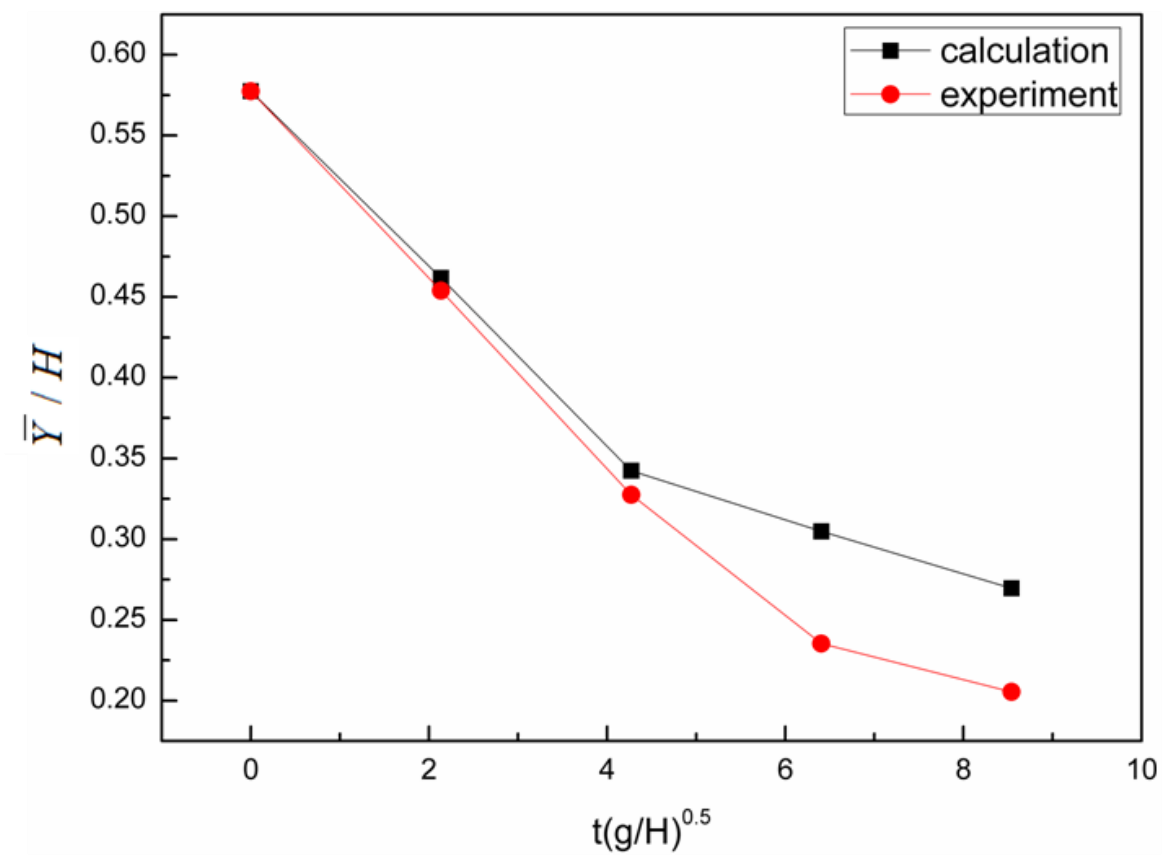

Fig.17 The relationship between the average vertical position of solid bodies and time

In Fig.17 and Fig.18, due to the reason above, the movement of solid bodies is larger than that in experiment, but in these two figures, an inflection point can be observed both in calculation and experiment, the movement of solid bodies shows the same tendency.

After comparing the simulation and the experiment of the breaking of a water dam involving solid cylinder layers, it can be concluded that the FOCUS code can do a good job in simulating the fluid-solid mixture flow. 


\subsection{Simulation of a three-dimensional fluid-solid mixture flow}

To verify the calculation of the situation in the three-dimension and be an attempt of the future work in the three-dimensional reactor core, a simple case about two solid bodies fall into water dam is simulated. In order to accelerate the computation, larger size and fewer particles are used, the size of the particle is set as $0.04 \mathrm{~m}$, each solid body contains 16 moving particles, and the total number of particles in this calculation is 6640 . The shape of solid bodies is set as cuboids, which means that the DEM model in this program can not only be used in the ship of sphere and circle. The density of the solid body is set as $500 \mathrm{~kg} / \mathrm{m}^{3}$. The initial particle arrangement is shown in Fig.19, the front panel of the container is hided to show the arrangement clearly.

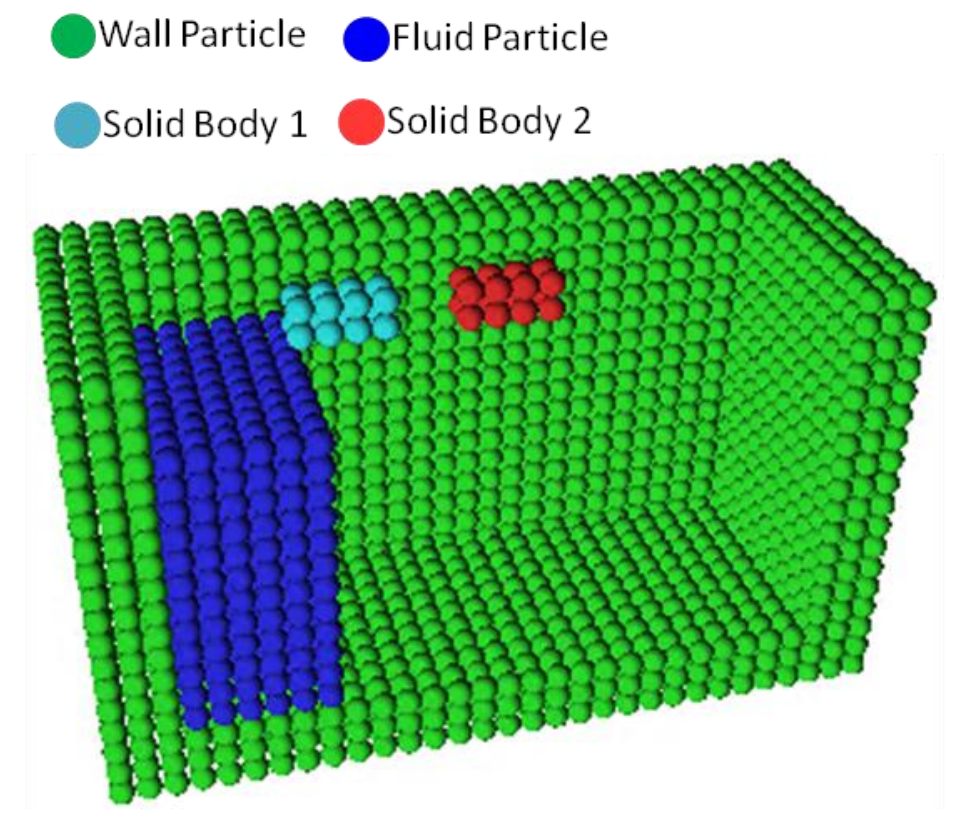

Fig.19 Initial particle arrangement of two solid bodies fall into water dam

The calculation results are shown in Fig.20, in order to observe the behaviors of two solid bodies, the movement of solid bodies is separately shown in Fig. 21 with the fluid particles hided, and the two solid bodies are distinguished by drawing in different color. As shown in Fig.20 and 21, solid bodies fall into the water dam at 0.3s, and reach the right wall of the container with water flow at $0.5 \mathrm{~s}$, then the right-most solid body is lift by the water wave at $0.7 \mathrm{~s}$, and two solid bodies change their positions by water at $1.0 \mathrm{~s}$.

Although there is no experiment for comparison, this three-dimensional calculation example shows the characteristic behaviors of the fluid-solid mixture flow, and the 
phenomenons shown in the calculation meet the common sense. It can be concluded by this simulation that the program has the capability of calculating the fluid-solid mixture flow in three-dimension.

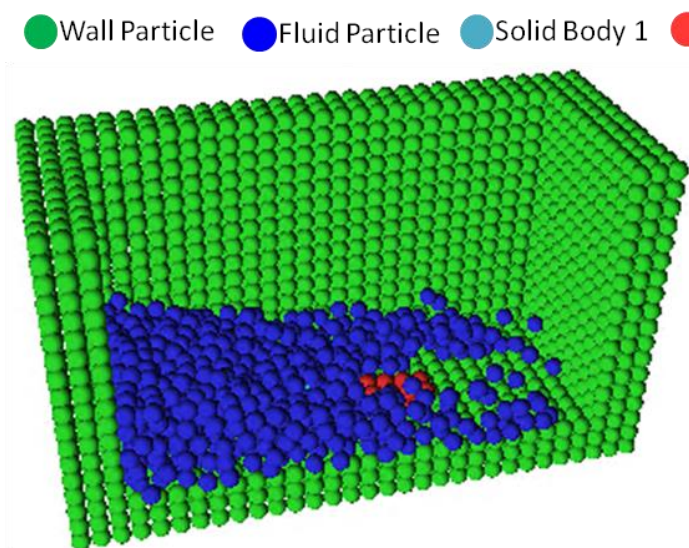

(a) $0.3 \mathrm{~s}$

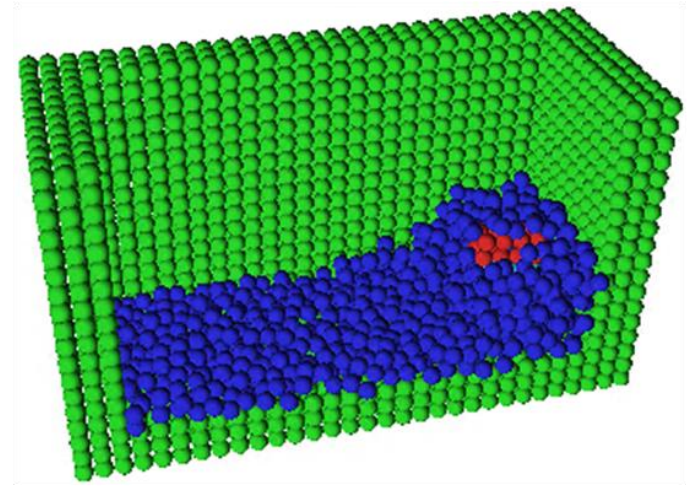

(c) $0.7 \mathrm{~s}$
Solid Body 2

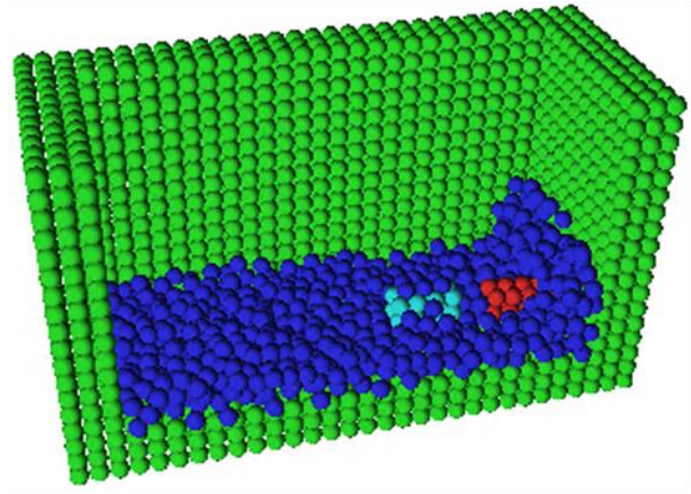

(b) $0.5 \mathrm{~s}$

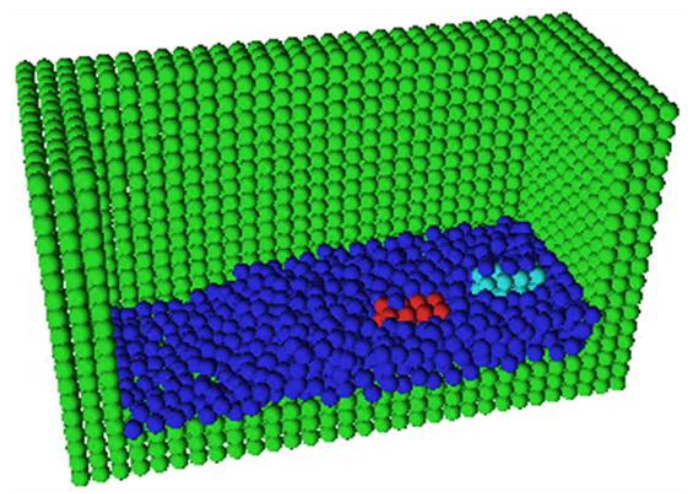

(d) $1.0 \mathrm{~s}$

Fig.20 Calculation results of two solid bodies fall into water dam

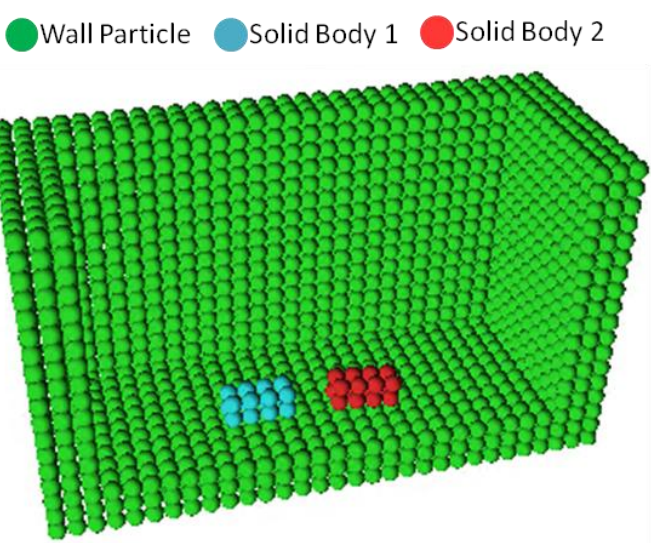

(a) $0.3 \mathrm{~s}$

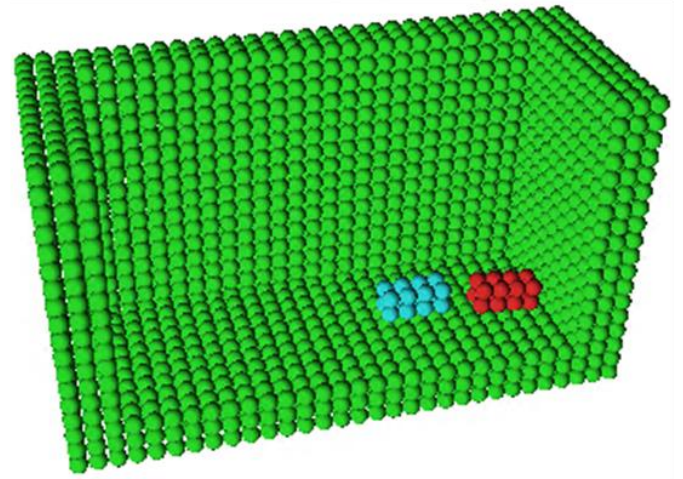

(b) $0.5 \mathrm{~s}$ 


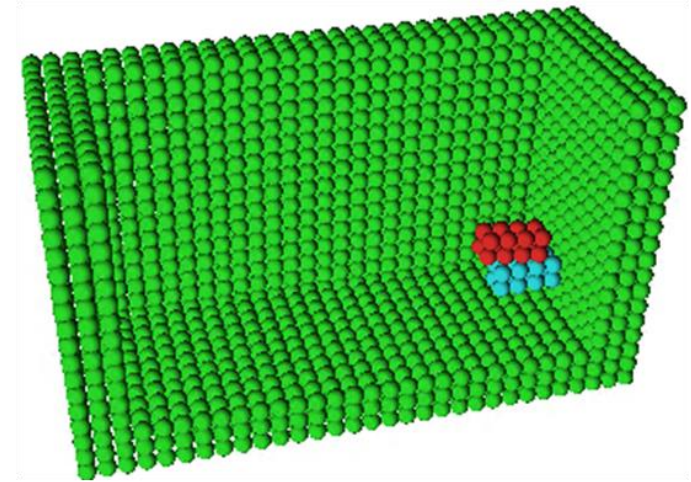

(c) $0.7 \mathrm{~s}$

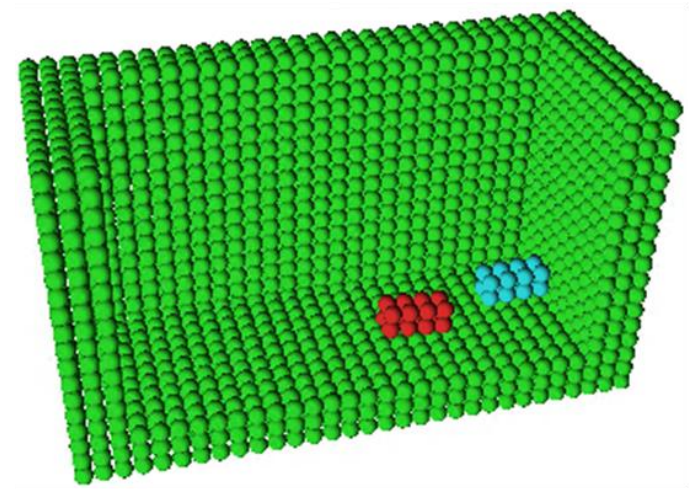

(b) $1.0 \mathrm{~s}$

Fig.21 Movement of solid bodies

\section{Conclusion}

In this study, the FOCUS code that can be applied to simulate the fluid-solid mixture flow is developed by coupling the discrete element method (DEM) with the moving particle semi-implicit (MPS) method. Both the models used in the MPS method and the numerical algorithm of DEM are introduced. Several simulations and experiments are carried out to validate the DEM model, PMS model and the interaction between fluid phase and solid phase. The calculation results and experiment data are in the good agreement. Furthermore, a simple three-dimensional case is simulated before the future work about severe accidents in reactor core. The parameters and time step in the DEM calculation are set artificially. In fact, the mechanical coefficients in the DEM calculation are determined by the elastic modulus and Poisson ratio material as shown in equations (28) to (33), and the time step of the DEM calculation is relative to the velocity of the Rayleigh wave. For the future studies, the equations of each coefficient and time step in the DEM calculation should be added into the FOCUS code.

There are still a lot of work should be done before applying the FOCUS code to analyze the severe accidents in the three-dimensional reactor core, because a series of physical chemical reaction between different materials will occurs in the melting process, and the molten corium always contains several components in different shapes. Thus, if the FOCUS code want to be adopted in simulating the severe accidents, the first problem should be solved is to simulate the collision between the irregular components. This process can be achieved by distinguishing the different collision radius in different 
direction of an irregular component, then the collision two components in different shapes can be calculated by DEM model. The detailed work will be carried out in the future.

\section{Acknowledgments}

The present study is supported by the National Science Foundation of China (no. 11505134) and by the China Postdoctoral Science Foundation (no. 2015M572568).

\section{References}

Koshizuka, 2010. Current Achievements and Future Perspectives on Particle Simulation Technologies for Fluid Dynamics and Heat Transfer, Journal of NUCLEAR SCIENCE and TECHNOLOGY, 48(2), 155-168.

Koshizuka S, Nobe A, Oka Y. Numerical analysis of breaking waves using the moving particle semi-implicit method[J]. International Journal for Numerical Methods in Fluids, 1998, 26(7):751-769.

Yoon H Y, Koshizuka S, Oka Y. Direct calculation of bubble growth, departure, and rise in nucleate pool boiling[J]. International Journal of Multiphase Flow, 2001, 27(2):277-298.

R.H. Chen, W.X. Tian, G.H. Su, et al., 2011. Numerical Investigation on Coalescence of Bubble Pairs Rising in a Stagnant Liquid, Chemical Engineering Science, 66(21), 5055-5063.

R.H. Chen, W.X. Tian, G.H. Su, et al.,2010. Numerical investigation on bubble dynamics during flow boiling using moving particle semi-implicit method, Nuclear Engineering and Design, 240(11), 3830-3840.

Duan G, Koshizuka S, Chen B. A contoured continuum surface force model for particle methods[J]. Journal of Computational Physics, 2015, 298(C):280-304.

R.H. Chen, Y. Oka, 2014a. Numerical analysis of freezing controlled penetration behavior of the molten core debris in an instrument tube with MPS, Annals of Nuclear Energy, 71, 382-398

R.H. Chen, Y. Oka, G. Li, et al., 2014b. Numerical investigation on melt freezing behavior in a tube by MPS method. Nuclear Engineering and Design, 273, 440-448.

Cundall P A., 1971. A compute model for simulating progressive large scale movement in blocky rock system, Proceedings of International Symponsor Rock Fracture, France: 
Nancy, II-8-II-12.

Zhang, L., Zhou, Y., Zhang, Y., Tian, W., Qiu, S., Su, G., 2015a. Natural convection heat transfer in corium pools: A review work of experimental studies. Prog Nucl Energ 79, 167-181.

Zhang, Y., Niu, S., Zhang, L., Qiu, S., Su, G., Tian, W., 2015b. A review on analysis of LWR severe accident. Journal of Nuclear Engineering and Radiation Science 1, 041018. Zhang, L., Zhang, Y., Zhao, B., Ma, W., Zhou, Y., Su, G.H., Tian, W., Qiu, S., 2016a. COPRA: A large scale experiment on natural convection heat transfer in corium pools with internal heating. Prog Nucl Energ 86, 138-140.

Zhang, L., Zhang, Y., Zhou, Y., Su, G.H., Tian, W., Qiu, S., 2016b. COPRA experiments on natural convection heat transfer in a volumetrically heated slice pool with high Rayleigh numbers. Annals of Nuclear Energy 87, Part 2, 81-88.

Koshizuka, S., Nobe, A. and Oka, Y., 1998. Numerical Analysis of Breaking Waves Using the Moving Particle Semi-implicit Method, International Journal for Numerical Method in Fluid, 26(7), 751-769.

Gotoh, H. and Sakai, T., 1997. Numerical Simulation of Sheetflow as Granular Material, Journal of Waterway, Port, Coastal and Ocean Engineering, 123(6), 389-396.

Gladwell G M L., 1980. Contact problem in the classical theory of elasticity. The Netherlands, 500-620.

Zhang, S., Morita, K., Shirakawa, N., Yamamoto, Y., 2009. Next Generation Safety Analysis Methods for SFRs-(4) Development of a Computational Framework on Fluid-Solid Mixture Flow Simulations for the COMPASS Code. International Conference on Nuclear Engineering , 58, 465-471. 